\title{
Health-Related Quality of Life Improvements in Systemic Lupus Erythematosus Derived from a Digital Therapeutic Plus Tele-Health Coaching Intervention: Randomized Controlled Pilot Trial
}

Faiz Khan ${ }^{1^{*}}$, MD; Nora Granville ${ }^{2^{*}}$, MA; Raja Malkani ${ }^{3^{*}}, \mathrm{BS}$; Yash Chathampally ${ }^{4^{*}}, \mathrm{MD}, \mathrm{MSc}$

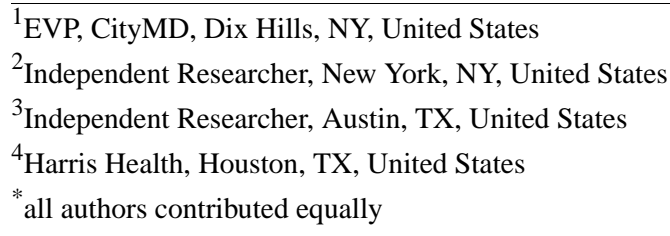

\section{Corresponding Author:}

Faiz Khan, MD

EVP, CityMD

101 Long Drive Court

Dix Hills, NY, 11746

United States

Phone: 15164269086

Email: fkhan@citymd.net

\begin{abstract}
Background: Systemic lupus erythematosus (SLE), a systemic autoimmune disease with no known cure, remains poorly understood and patients suffer from many gaps in care. Recent work has suggested that dietary and other lifestyle factors play an important role in triggering and propagating SLE in some susceptible individuals. However, the magnitude of influence of these triggers, how to identify pertinent triggers in individual patients, and whether removing these triggers confers clinical benefit is unknown.
\end{abstract}

Objective: To demonstrate that a digital therapeutic intervention, utilizing a mobile app that allows self-tracking of dietary, environmental, and lifestyle triggers, paired with telehealth coaching, added to usual care, improves quality of life in patients with SLE compared with usual care alone.

Methods: In this randomized controlled pilot study, adults with SLE were assigned to a 16-week digital therapeutic intervention plus usual care or usual care alone. Primary outcome measures were changes from baseline to 16 weeks on 3 validated health-related quality of life (HRQoL) tools: Functional Assessment of Chronic Illness Therapy-Fatigue (FACIT-F), Brief Pain Inventory-Short Form (BPI-SF), and Lupus Quality of Life (LupusQoL).

Results: A total of 50 patients were randomized ( 23 control, 27 intervention). In per-protocol analysis, the intervention group achieved significantly greater improvement than the control group in 9 of 11 domains: FACIT-F ( $34 \%$ absolute improvement for the intervention group vs $-1 \%$ for the control group, $P<.001$ ), BPI-SF-Pain Interference (25\% vs $0 \%, P=.02$ ), LupusQoL-Planning (17\% vs $0 \%, P=.004)$, LupusQoL-Pain ( $13 \%$ vs $0 \%, P=.004)$, LupusQoL-Emotional Health (21\% vs $4 \%, P=.02)$, and LupusQoL-Fatigue (38\% vs 13\%, $P<.001$ ) were significant when controlling for multiple comparisons; BPI-SF-Pain Severity (13\% vs $-6 \%, P=.049$ ), LupusQoL-Physical Health (17\% vs 3\%, $P=.049)$, and LupusQoL-Burden to Others (33\% vs 4\%, $P=.04$ ) were significant at an unadjusted 5\% significance level.

Conclusions: A digital therapeutic intervention that pairs self-tracking with telehealth coaching to identify and remove dietary, environmental, and lifestyle symptom triggers resulted in statistically significant, clinically meaningful improvements in HRQoL when added to usual care in patients with SLE.

Trial Registration: ClinicalTrials.gov NCT03426384; https://clinicaltrials.gov/ct2/show/NCT03426384

(J Med Internet Res 2020;22(10):e23868) doi: 10.2196/23868 


\section{KEYWORDS}

systemic lupus erythematosus; digital health; digital therapeutic; autoimmunity; food as medicine; dietary intervention; health-related quality of life; lifestyle medicine; mobile health; environmental influences on autoimmunity

\section{Introduction}

\section{Background}

Systemic lupus erythematosus (SLE) is a multisystem, complex autoimmune disease of unclear etiology affecting at least 1.5 million Americans and 5 million worldwide [1]. The hallmark of the disease is uncontrolled inflammation in otherwise healthy tissue which can lead to organ damage and sometimes even organ failure. SLE can affect any body system-the kidneys, skin, joints, heart, lungs, gastrointestinal system, and nervous system may all become involved, and the pattern of involvement differs from patient to patient. The most common symptoms are fever, rash, profound fatigue, and joint pain and swelling. Disease activity is prone to exacerbations (called flares) alternating with periods of remission in cycles that are often unpredictable and therefore have an even more detrimental effect on quality of life.

There is no cure for SLE and universally effective treatment is not available. Current management relies on immune modulating drugs, but their side effects often increase discomfort and their use carries the risk of severe adverse events [2]. While 5-year survival rates have increased dramatically from $50 \%$ to $90 \%$ $[3,4]$, patients with SLE still have significantly higher age-standardized mortality rates [5] and lower health-related quality of life (HRQoL) than the general population [6]. Underemployment and work disability, associated primarily with fatigue and pain, are common [7,8]. Young women are disproportionately affected, especially those of non-Caucasian ethnicity and low socioeconomic status. It has recently been reported that SLE is the leading cause of death among chronic inflammatory diseases in women aged 15-24, with death rates exceeding those of HIV and diabetes [9].

Although a clear understanding of the pathophysiology of SLE remains elusive, the recognition that an individual's DNA blueprint alone does not wholly account for disease occurrence has fueled new areas of research into the environmental and lifestyle determinants of SLE. Important to this line of inquiry is (1) the growing recognition that epigenetic alterations, such as DNA methylation, noncoding RNAs, and histone modifications, are involved in the development of autoimmune diseases [10] and (2) emerging evidence from human and animal studies that these epigenetic processes are influenced by dietary, environmental, and lifestyle factors [11-23]. Modifying these factors presents an attractive, low-risk treatment option for SLE. However, there is much work to be done to better define these potential triggers and determine if eliminating them confers clinical benefit. Complicating these efforts is the fact that SLE is an extremely heterogeneous disease. Widely variable initial presentation, disease course, organ involvement, and response to treatment complicate diagnosis, management, and clinical research efforts. An international team of experts have identified SLE heterogeneity as "the primary barrier hindering advancement" [24]. Given this heterogeneity, it is reasonable to hypothesize that numerous dietary, environmental, and lifestyle SLE triggers exist and differ from patient to patient. Therefore, tracking and analyzing possible trigger-symptom associations require a reliable, accurate, easy-to-use method for gathering and processing a considerable amount of data. Digital therapeutics can accomplish this and may offer unique solutions to the obstacles faced in attempts to address the dietary, environmental, and lifestyle triggers of SLE.

Several digital therapeutics have already received FDA clearance, and many more are in development, to address a range of medical conditions, including prediabetes and diabetes, substance use and opioid use disorders, Alzheimer disease, obesity, hypertension, chronic back pain, attention-deficit/hyperactivity disorder, concussion, and multiple sclerosis [25-27]. Patients with SLE can use several available apps to help track symptoms and disease activity [28-30] and manage medications [31]. However, none of the existing apps have successfully addressed the relationship between dietary, environmental, and lifestyle factors and symptom severity in autoimmunity.

A digital therapeutic platform has been developed which combines self-tracking technology, analytics, and tele-health coaching to identify and remove possible dietary, environmental, or lifestyle triggers, with the goal to provide clinically meaningful improvements in symptoms and HRQoL in those with autoimmune disease. The platform is intended as an adjunct to standard of care.

The platform was developed over several years with extensive feedback from stakeholders in the autoimmune disease community. This has included discussions with patients, family members, physicians, insurance providers, foundations, patient advocacy groups, pharmaceutical companies, and even potential service providers with experience in the sector, such as contract research organizations. The goal has been to commercialize a product that serves an unmet clinical need, but also that fits into the clinical workflow, would be widely adopted, and has a pathway to reimbursement. As a digital therapeutic, the product is also able to track patient usage and engagement during the course of the program, and notifications can be sent following the program to track longer-term outcomes.

Usability and patient preferences have been carefully considered to ensure that a broad range of individuals are comfortable engaging with the smartphone interface, participating in coaching sessions, and complying with suggested interventions throughout the program. Similarly, the web portal and the health coaching protocol itself were iteratively refined through consultation with health coaches and health care providers.

This novel approach is unique in that it implicitly takes disease heterogeneity into account, leverages the growing understanding of the role environment plays in initiating and propagating autoimmune disease, and personalizes each patient's recommendations based on software data analytics. 


\section{Objective}

The objective of this study was to determine whether the addition to usual care of this digital therapeutic program-intended to identify and intervene on dietary and other lifestyle factors found by data analytics to be associated with symptom frequency and severity-improved HRQoL in patients with SLE more than usual care alone.

\section{Methods}

\section{Study Population}

The study enrolled adults ( $\geq 18$ years) across the United States from December 2017 to May 2018. Participants were recruited through the following online forums: Lupus Friends and Family, Flare Fighter, and Purple Wings Facebook groups; and Clara Health and Autoimmune Registry (online resources for patients interested in participating in clinical trials). Interested individuals completed a prequalification survey online, and only those individuals who passed the prequalification survey (ie, those who were not disqualified) were asked to submit medical records which were reviewed by the study principal investigator (FK) to verify a diagnosis of SLE and confirm all inclusion/exclusion criteria. Eligible individuals underwent a phone consent session and electronically signed an informed consent document if they chose to participate. Participants were assigned to either the intervention or control arm via randomized blocks of 3 to 8 individuals using a cryptographic random seed, targeting 1:1 allocation between groups. After randomization and electronic collection of baseline data, randomization groups were made known to participants.
Inclusion criteria included owning a smartphone, a threshold score for at least one of seven pain and fatigue questions, and taking a stable dose of one of more of the following drugs for 3 or more months prior to study enrollment: immunosuppressive or immunomodulating therapy (biologic or nonbiologic), immunoglobulin therapy, or $20 \mathrm{mg}$ or more of prednisone (or equivalent corticosteroid). Exclusion criteria included pre-existing or incident diagnosis of cancer, pregnancy, or intention to conceive during the study period, and criteria intended to avoid confounding interpretation of changes in outcome: current or planned participation in another interventional or observational study, known plans to alter inclusion criteria medications prior to onset of study or during study, and use of pulse steroids for more than 30 days combined or during the last 4 weeks of the intervention period.

The occurrence of adverse events was assessed during the coaching sessions and by a call to every participant by the principal investigator at the conclusion of the study. Coaches were instructed to convey possible adverse events to a study team physician (FK) who would report them to the Institutional Review Board.

The study was approved by Western Institutional Review Board (CSI: NCT03426384).

\section{Intervention}

The digital therapeutic technology has 3 key components: a smartphone iOS or Android app for the patient to track lifestyle activities (eg, diet, sleep habits, physical activity, bowel movements) and symptoms (Figure 1); software that analyzes and organizes data; and a web portal that presents all patient data to the health coach. 
Figure 1. Mobile app for entering symptoms and dietary, environmental, and lifestyle inputs.

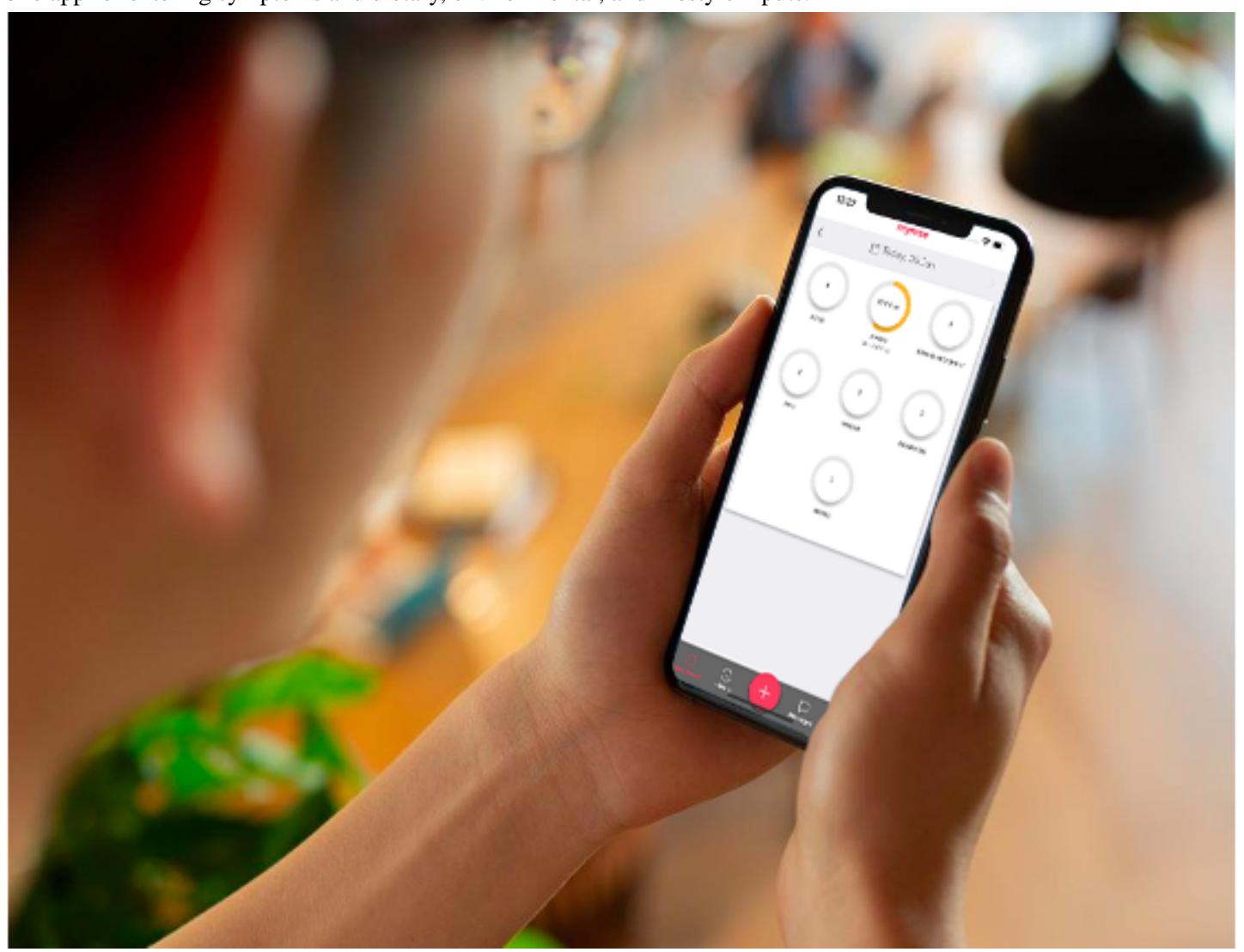

During weekly telehealth coaching sessions, the health coach viewed the data in the web portal and recommended interventions intended to confirm or reject suspected dietary, environmental, and lifestyle triggers. Potential trigger-symptom correlations were presented by the software and the prioritization of triggers and the decision on which intervention to suggest were performed by the health coach. Throughout the program, tracked symptoms were not a comprehensive representation of all participant's complaints but rather were chosen by the coach based on their propensity to fluctuate, thereby providing indicators which were more likely to be responsive to change. The health coach also provided nutrition and lifestyle education as needed (eg, addition of protein at breakfast, instruction on nutrient-dense foods, recommendations for stress management techniques). Over-the-counter supplementation was recommended by the health coach on a case-by-case basis as appropriate (eg, vitamin D3 if patient records showed deficiency that had not already been addressed-see Multimedia Appendix 1 for full details). A single, certified health coach performed all coaching sessions for every participant. Participants randomized to the intervention group received an email with instructions on how to download the app, create a profile, and use the app to track dietary input. Throughout the study period, participants could receive technology and other support by messaging (through the app) or emailing the study team at any time. After 3-5 days of taking pictures of all the food and beverages consumed daily, participants completed an introductory telephone session to identify their symptoms and goals of the program, review initial tracking data, and receive further training on tracking of other environmental and lifestyle inputs.
Following this initial call, weekly 20-30-minute telehealth coaching sessions were scheduled for the ensuing 15 weeks. Each week, based on associations presented by the software between dietary or other tracked exposures (triggers) and symptoms, the coach suggested behavioral interventions to ameliorate symptoms (eg, eliminate dairy if a patient's joint pain appeared to flare in relation to dairy intake over the past week). The results of these iterative, weekly interventions were reviewed in subsequent sessions. Compliance with interventions was assessed by analysis of digital tracking and weekly coaching discussions. Successful interventions were maintained, whereas those which did not impact symptoms were either rejected or subjected to longer trials.

Control group participants continued usual care as recommended by their treating physician(s); were not introduced to the intervention app (or any other sham app); and received no training, coaching, or other study interventions.

Prior to entry into the study, all participants had a call with a study staff member to review a summary of the trial, the intervention procedures and schedule, potential risks and benefits, alternative treatments, and provide informed consent. Control participants completed the same battery of assessments at the same intervals as the intervention group participants. At the end of 16 weeks, control participants met with a study team member by phone during which time final assessment surveys were administered, adverse events over the prior 16 weeks were ascertained, and the opportunity for cross-over to receive the digital therapeutic intervention was offered. All surveys were completed via an HIPAA (Health Insurance Portability and Accountability Act)-compliant version of SurveyGizmo. 


\section{Outcomes}

The primary outcomes were changes between baseline and week 16 in 11 domains reflecting various aspects of HRQoL, as assessed by 3 validated patient-reported outcome measures (PROMs): (1) Functional Assessment of Chronic Illness Therapy-Fatigue (FACIT-F), consisting of 13 questions aggregated into 1 domain measuring fatigue; (2) Brief Pain Inventory-Short Form (BPI-SF), consisting of 15 questions classified into 2 domains (pain severity and pain interference), and; (3) Lupus Quality of Life (LupusQoL), consisting of 34 questions classified into 8 domains (fatigue, physical health, planning, burden to others, emotional health, pain, intimate relationships, and body image). All 3 outcome measures have been previously described [32-34] and validated for use in SLE $[29,35,36]$. The participants were asked to complete these PROMs on a secure website prior to the start of the intervention and at weeks 4, 8, 12, and 16 .

Secondary outcomes (derived from analysis of tracking data and coach dashboard information) were tracking adherence (the number of days a participant logged at least one observation into the mobile app in a 24-hour period); session adherence (the number of weekly coaching calls a participant participated in over the 16 weeks); and types and prevalence of (1) the most commonly tracked symptoms, (2) suspected triggers, and (3) interventions. These data were generated from the participant's tracking data and coaching notes.

\section{Adherence}

Tracking adherence was calculated as the number of days (24-hour period) at least one observation (eg, symptom, food, other lifestyle input) was entered into the app divided by the number of days in the 16 -week program $(n=112)$ to arrive at the percentage of days with tracked data (adherence of $100 \%$ indicates that the participant used the app to track more than once/day each day of the program). Coaching session adherence was calculated as the number of coaching sessions completed by the participant divided by 16 and converted to a percentage (adherence of $100 \%$ indicates that the participant completed 1 or more session/week each week of the program). Median and 25th and 75th percentile values for tracking and session adherence were then calculated for the whole group.

\section{Statistical Analysis}

Prior to the study, the sample size was computed based on the Mann-Whitney $U$ test to provide approximately $80 \%$ power to detect an effect size proportional to a mean difference in improvement of about $10 \%$ with a standard deviation of $10 \%$ without correcting for multiple comparisons. This effect size was chosen based on early user experience with the program as well as consideration of previously established minimally important differences for the outcome measures [35-37]. It was determined that a sample size of 50 was sufficient to allow for attrition and still produce the needed power with the remaining participants expected to complete the study. To balance minimizing type I and type II errors, results were highlighted that were significant at an unadjusted significance level of 5\% and also, due to the high level of correlation in outcomes, at a level adjusted using the Benjamini-Hochberg method to control the false detection rate at $5 \%$ for multiple comparisons.

Nonparametric tests were chosen based on minimal distributional assumptions given the small sample size: Wilcoxon signed-rank test for the change within the intervention and control groups between the baseline and 16-week/end-of-program domain scores; and Mann-Whitney $U$ test for changes in score between the intervention and control groups. Medians, 25th, and 75th percentile values are displayed as measures of central tendency and spread. All statistical analyses were performed using IBM SPSS Statistics Subscription (Build 1.0.0.1072).

Intention-to-treat (ITT) analysis included participants who met inclusion criteria at the start of the intervention period, even if they did not complete the study. Per-protocol (PP) analysis was limited to participants who completed 10 or more sessions within the 16-week study period (based on prior exploratory testing), submitted end-of-study data, and experienced no exclusions. Missing follow-up scores from participants who dropped out of the intervention group were populated with the worst observed scores for that time point, thus biasing toward the null hypothesis.

\section{Results}

\section{Study Population}

In total, 50 patients were enrolled, with 47 included in ITT analysis and 34 in PP analysis (Figure 2). Table 1 shows the study population baseline demographics. The control and intervention groups were similar across most categories and any differences were not expected to impact results. For the full cohort, the median age was $43 ; 96 \%$ (44/46) were female; $59 \%$ (27/46) of participants were Caucasian, 17\% (8/46) Black or African American, and 24\% (11/46) Hispanic. Of the 25 ITT intervention participants, 6 (24\%) were lost to follow up after completing 0 or 1 coaching sessions ( 1 discontinued inclusion medication after 1 session; 1 voluntarily withdrew after 1 session to care for a sick family member; 4 were lost to follow up after completing 1 [ $n=3$ participants] or no $[n=1]$ coaching sessions). Of the remaining 19, 16 completed at least ten coaching sessions over 16 weeks (for a completion rate of $84 \%$ ) and were included in PP analysis. Medications at study entry and baseline scores on the 3 PROMs are shown in Table 2. 
Figure 2. Participant flow. ITT: intention to treat; PP: per protocol.

\section{PARTICIPANT FLOW}

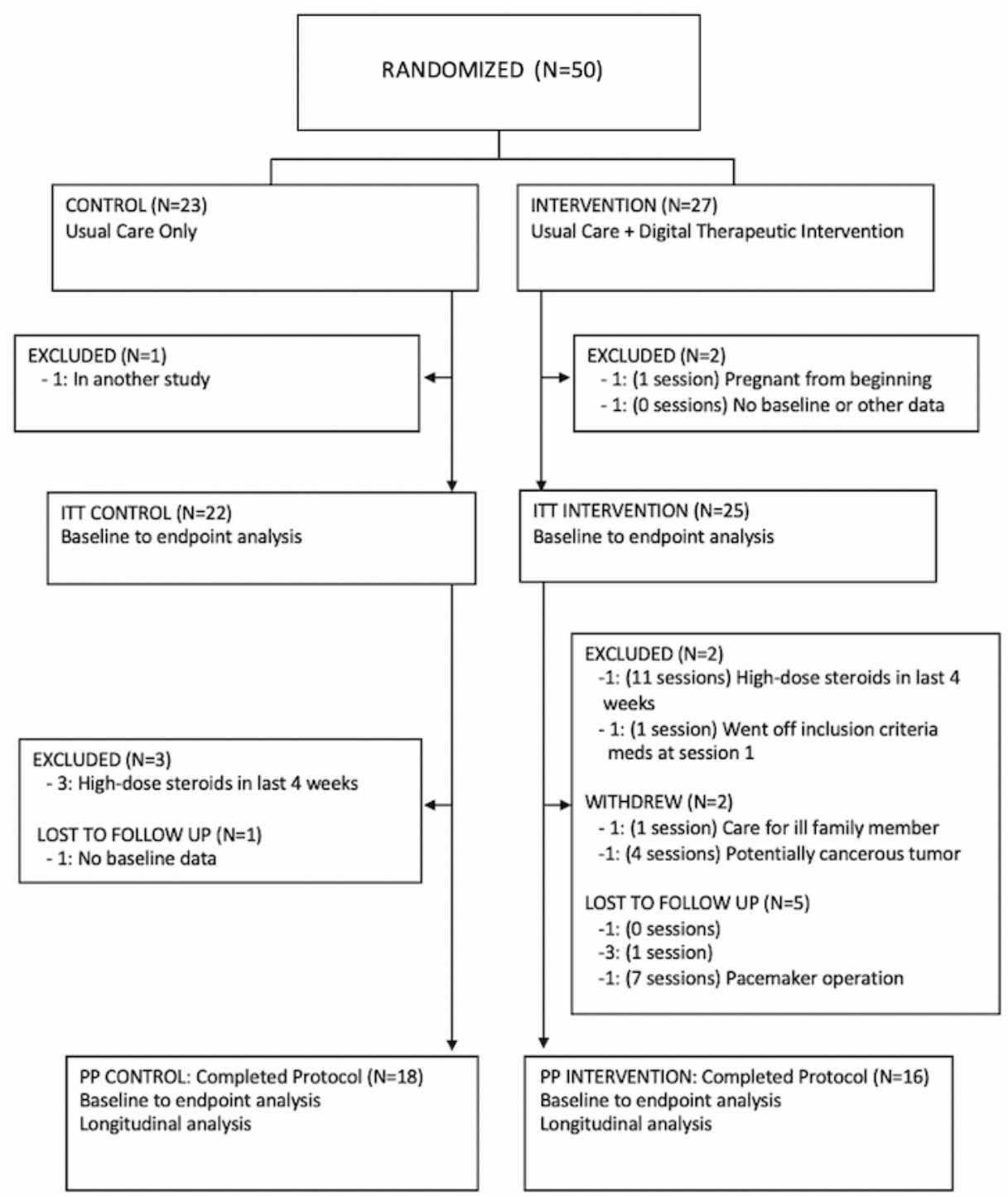


Table 1. Study population demographics. ${ }^{\mathrm{a}}$

\begin{tabular}{|c|c|c|c|c|c|c|}
\hline \multirow[t]{2}{*}{ Demographics } & \multicolumn{2}{|l|}{$\mathrm{ITT}^{\mathrm{b}}$} & \multicolumn{2}{|l|}{$\mathrm{PP}^{\mathrm{c}}$} & \multicolumn{2}{|l|}{$\mathrm{EPP}^{\mathrm{d}}$} \\
\hline & Control & Intervention & Control & Intervention & Control & Intervention \\
\hline Participants who provided baseline data, $\mathrm{N}$ & 21 & 25 & 18 & 16 & 3 & 9 \\
\hline Age, median (25th percentile, 75 th percentile) & $42(36,50)$ & $44(33,51)$ & $42(35,50)$ & $45(35,54)$ & $43(37,59)$ & $36(31,47)$ \\
\hline \multicolumn{7}{|l|}{ Ethnic background } \\
\hline Black or African American & $2(10)$ & $6(24)$ & $2(11)$ & $3(19)$ & $0(0)$ & $3(33)$ \\
\hline Caucasian or White & $12(57)$ & $15(60)$ & $9(50)$ & $10(63)$ & $3(100)$ & $5(56)$ \\
\hline Hispanic or Latino & $7(33)$ & $4(16)$ & $7(39)$ & $3(19)$ & $0(0)$ & $1(11)$ \\
\hline \multicolumn{7}{|l|}{ Primary language } \\
\hline English & $20(95)$ & $25(100)$ & $17(94)$ & $16(100)$ & $3(100)$ & $9(100)$ \\
\hline Spanish & $1(5)$ & $0(0)$ & $1(6)$ & $0(0)$ & $0(0)$ & $0(0)$ \\
\hline \multicolumn{7}{|l|}{ Gender } \\
\hline Female & $20(95)$ & $24(96)$ & $17(94)$ & $15(94)$ & $3(100)$ & $9(100)$ \\
\hline Male & $1(5)$ & $1(4)$ & $1(6)$ & $1(6)$ & $0(0)$ & $0(0)$ \\
\hline \multicolumn{7}{|l|}{ Education level } \\
\hline Some high school & $0(0)$ & $1(4)$ & $0(0)$ & $0(0)$ & $0(0)$ & $1(11)$ \\
\hline High school & $1(5)$ & $0(0)$ & $1(6)$ & $0(0)$ & $0(0)$ & $0(0)$ \\
\hline Some college/trade/technical training & $4(19)$ & $7(28)$ & $3(17)$ & $3(19)$ & $1(33)$ & $4(44)$ \\
\hline Associate degree & $5(24)$ & $6(24)$ & $4(22)$ & $5(31)$ & $1(33)$ & $1(11)$ \\
\hline Bachelor's degree & $9(43)$ & $5(20)$ & $8(44)$ & $3(19)$ & $1(33)$ & $2(22)$ \\
\hline Master's/Professional degree & $2(10)$ & $6(24)$ & $2(11)$ & $5(31)$ & $0(0)$ & $1(11)$ \\
\hline \multicolumn{7}{|l|}{ Employment } \\
\hline Full-time paid & $7(33)$ & $7(28)$ & $7(39)$ & $5(31)$ & $0(0)$ & $2(22)$ \\
\hline Part-time paid & $3(14)$ & $4(16)$ & $3(17)$ & $3(19)$ & $0(0)$ & $1(11)$ \\
\hline Self-employed & $1(5)$ & $1(4)$ & $0(0)$ & $1(6)$ & $1(33)$ & $0(0)$ \\
\hline Homemaker & $1(5)$ & $0(0)$ & $1(6)$ & $0(0)$ & $0(0)$ & $0(0)$ \\
\hline Out of work, not currently looking & $0(0)$ & $1(4)$ & $0(0)$ & $1(6)$ & $0(0)$ & $0(0)$ \\
\hline Unable to work—on disability & $5(24)$ & $11(44)$ & $3(17)$ & $6(38)$ & $2(67)$ & $5(56)$ \\
\hline Unable to work—other & $4(19)$ & $1(4)$ & $4(22)$ & $0(0)$ & $0(0)$ & $1(11)$ \\
\hline \multicolumn{7}{|l|}{ Income Level } \\
\hline US $\$ 0$-US $\$ 25,999$ & $6(29)$ & $7(28)$ & $6(33)$ & $3(19)$ & $0(0)$ & $4(44)$ \\
\hline US $\$ 26,000-$ US $\$ 51,999$ & $5(24)$ & $7(28)$ & $3(17)$ & $5(31)$ & $2(67)$ & $2(22)$ \\
\hline US $\$ 52,000-$ US $\$ 74,999$ & $3(14)$ & $6(24)$ & $2(11)$ & $4(25)$ & $1(33)$ & $2(22)$ \\
\hline More than US $\$ 75,000$ & $7(33)$ & $5(20)$ & $7(39)$ & $4(25)$ & $0(0)$ & $1(11)$ \\
\hline \multicolumn{7}{|l|}{ Relationship status } \\
\hline Life partner (married/other) & $13(62)$ & $15(60)$ & $11(61)$ & $11(69)$ & $2(67)$ & $4(44)$ \\
\hline Single/separate/divorced/widowed & $8(38)$ & $10(40)$ & $7(39)$ & $5(31)$ & $1(33)$ & $5(56)$ \\
\hline
\end{tabular}

${ }^{\mathrm{a}}$ Values are numbers (percentages) unless stated otherwise.

bITT: intention to treat.

${ }^{\mathrm{c}}$ PP: per protocol.

${ }^{\mathrm{d}}$ EPP: ITT participants who were excluded from PP. 
Table 2. Study population inclusion medications and baseline patient-reported outcome measure scores. ${ }^{\text {a }}$

\begin{tabular}{|c|c|c|c|c|c|c|}
\hline \multirow[t]{2}{*}{ Medications and outcome measure scores } & \multicolumn{2}{|l|}{$\mathrm{ITT}^{\mathrm{b}}$} & \multicolumn{2}{|l|}{$\mathrm{PP}^{\mathrm{c}}$} & \multicolumn{2}{|l|}{$\mathrm{EPP}^{\mathrm{d}}$} \\
\hline & Control & Intervention & Control & Intervention & Control & Intervention \\
\hline Participants who provided baseline data, $\mathrm{N}$ & 21 & 25 & 18 & 16 & 3 & 9 \\
\hline \multicolumn{7}{|l|}{ Inclusion medications ${ }^{\mathrm{e}}$} \\
\hline Azathioprine & $2(10)$ & $5(20)$ & $1(6)$ & $1(6)$ & $1(33)$ & $4(44)$ \\
\hline Belimumab & $4(19)$ & $7(28)$ & $3(17)$ & $4(25)$ & $1(33)$ & $3(33)$ \\
\hline Hydroxychloroquine & $17(81)$ & $19(76)$ & $13(72)$ & $13(81)$ & $3(100)$ & $6(67)$ \\
\hline Immunoglobulin infusions & $1(5)$ & $0(0)$ & $1(6)$ & $0(0)$ & $0(0)$ & $0(0)$ \\
\hline Leflunomide & $0(0)$ & $1(4)$ & $0(0)$ & $1(6)$ & $0(0)$ & $0(0)$ \\
\hline Methotrexate & $4(19)$ & $3(12)$ & $4(22)$ & $2(13)$ & $0(0)$ & $1(11)$ \\
\hline Mycophenolate mofetil & $5(24)$ & $2(8)$ & $5(28)$ & $2(13)$ & $0(0)$ & $0(0)$ \\
\hline \multicolumn{7}{|c|}{ HRQoL ${ }^{\mathrm{f}}$, median (25th percentile, 75 th percentile) } \\
\hline FACIT $^{\mathrm{g}}$-Fatigue & $20(14,26)$ & $16(10,23)$ & $20(14,27)$ & $20(10,26)$ & $7(4,18)$ & $13(8,19)$ \\
\hline BPI-SF ${ }^{\mathrm{h}}$-Pain Severity & $4(3,5.7)$ & $5(3,6)$ & $3(3,5)$ & $4(3,6)$ & $5(5,7)$ & $6(3,7)$ \\
\hline BPI-SF-Pain Interference & $5(5,6)$ & $6(4,7)$ & $5(4,6)$ & $6(4,7)$ & $7(3,7)$ & $6(6,8)$ \\
\hline LupusQoL ${ }^{\mathrm{i}}$-Physical Health & $50(25,59)$ & $46(28,56)$ & $51(28,65)$ & $51(34,60)$ & $25(15,28)$ & $25(12,50)$ \\
\hline LupusQoL-Pain & $41(16,66)$ & $41(25,66)$ & $58(25,75)$ & $50(33,66)$ & $16(8,33)$ & $25(8,50)$ \\
\hline LupusQoL-Planning & $50(25,75)$ & $41(8,66)$ & $62(25,75)$ & $62(25,75)$ & $41(0,41)$ & $25(8,25)$ \\
\hline LupusQoL-Intimate Relationships & $50(25,87)$ & $56(25,75)$ & $50(25,75)$ & $75(25,81)$ & $68(50,87)$ & $31(25,50)$ \\
\hline LupusQoL-Burden to Others & $25(0,41)$ & $16(0,41)$ & $25(0,58)$ & $25(4,50)$ & $0(0,33)$ & $16(0,16)$ \\
\hline LupusQoL-Emotional Health & $54(37,70)$ & $54(29,66)$ & $56(37,70)$ & $60(35,79)$ & $50(20,70)$ & $20(16,54)$ \\
\hline LupusQoL-Body Image & $37(20,56)$ & $50(25,69)$ & $31(20,65)$ & $65(18,75)$ & $40(35,50)$ & $31(25,45)$ \\
\hline LupusQoL-Fatigue & $25(6,37)$ & $25(18,31)$ & $25(12,43)$ & $28(25,50)$ & $6(0,31)$ & $18(0,18)$ \\
\hline
\end{tabular}

${ }^{\mathrm{a}}$ Values are $\mathrm{n}(\%)$ unless stated otherwise.

${ }^{\mathrm{b}}$ ITT: intention to treat.

${ }^{\mathrm{c}} \mathrm{PP}$ : per protocol.

${ }^{\mathrm{d}}$ EPP: ITT participants who were excluded from PP.

${ }^{\mathrm{e}}$ Totals do not equal $100 \%$ as many patients were on multiple medications.

${ }^{\mathrm{f}} \mathrm{HRQoL}$ : health-related quality of life.

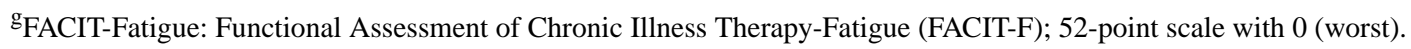

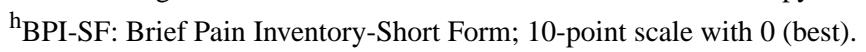

${ }^{\mathrm{i}}$ LupusQoL: Lupus Quality of Life; 100-point scale with 0 (worst).

\section{Adherence}

Table 3 shows tracking and coaching session adherence for the ITT and PP groups. In each group, tracking adherence exceeded $90 \%$ and coaching session adherence exceeded $80 \%$. In the ITT group, 16/25 (64\%) and 14/25 (56\%) participants reached greater than $70 \%$ tracking and session adherence, respectively. In the
PP group, 16/16 (100\%) and 13/16 (81\%) participants reached greater than $70 \%$ tracking and session adherence, respectively. The percentage of participants achieving $70 \%$ or greater tracking and session adherence is reported based on early experience with the platform indicating that this level of engagement correlates with better outcomes.

Table 3. Adherence results.

\begin{tabular}{lllll}
\hline Adherence & $\begin{array}{l}\text { Intention to treat } \\
\text { Tracking } \%\end{array}$ & Coaching sessions \% & $\begin{array}{l}\text { Per protocol } \\
\text { Tracking \% }\end{array}$ & Coaching sessions \% \\
\hline Median (25th, 75th percentile) & $91.1(50.9,97.3)$ & $81.3(25.0,81.3)$ & $96.9(94.4,99.1)$ & $81.3(81.3,93.8)$ \\
Over 70\% adherence, $\mathrm{n} / \mathrm{N}(\%)$ & $16 / 25(64)$ & $14 / 25(56)$ & $16 / 16(100)$ & $13 / 16(81)$ \\
\hline
\end{tabular}




\section{Intention-to-Treat Analysis}

Within the intervention group, significant improvement over baseline was noted for FACIT-F (median of 26.0 at the end of study vs 16.0 baseline, $P=.04$ ), LupusQoL-Burden to Others (25.0 vs $16.7, P=.02$ ), and LupusQoL-Fatigue (62.5 vs 25.0 , $P=.007)$. Within the control group, LupusQoL-Burden to Others (41.7 vs $20.8, P=.04$ ), LupusQoL-Body Image (45.0 vs 35.0 , $P=.047$ ), and LupusQoL-Fatigue (31.3 vs $25.0, P=.03$ ) saw improvement over baseline at 16 weeks. Comparing the 2 groups, although the intervention group improved more than the control group in 6 of 11 domains (FACIT-F, BPI-SF-Pain interference, LupusQoL-Pain, LupusQoL-Emotional Health, LupusQoL-Body Image, and LupusQoL-Fatigue), none of these comparisons reached statistical significance (Table 4). No significant improvements were uncovered when the Benjamini-Hochberg adjustment was applied to the significance level to account for multiple comparisons. 
Table 4. Intention-to-treat analysis of change in FACIT, BPI-SF, and LupusQoL domain scores from baseline to end of program. ${ }^{\text {a }}$

\begin{tabular}{|c|c|c|c|c|c|c|c|}
\hline \multirow[t]{2}{*}{ Domain } & \multicolumn{3}{|c|}{ Within group } & & \multicolumn{3}{|c|}{ Between group } \\
\hline & Count & Baseline & $\begin{array}{l}\text { End of program } \\
\text { (EOP) }\end{array}$ & Change in score (EOP: Baseline) & $P$-value & Difference $^{b}$ & $P$-value \\
\hline
\end{tabular}

FACIT $^{c}$-Fatigue (range 0-52, higher is better)

$\begin{array}{lllll}\text { Intervention } & 25 & 16.0(9.5,23.5) & 26.0(4.0,44.0) & 4.0(-3.5,21.0) \\ \text { Control } & 22 & 19.5(7.0,26.3) & 21.0(10.5,28.3) & -0.5(-5.0,7.3)\end{array}$

BPI-SF $^{d}$-Pain Severity (range 0-10, lower is better)

$\begin{array}{llll}\text { Intervention } & 25 & 5.3(3.0,6.8) & 5.3(2.1,8.3) \\ \text { Control } & 22 & 4.5(3.0,6.6) & 4.4(2.6,7.1)\end{array}$

BPI-SF-Pain Interference (range 0-10, lower is better)

$\begin{array}{llll}\text { Intervention } & 25 & 6.4(4.4,7.9) & 4.7(1.6,9.3) \\ \text { Control } & 22 & 5.6(4.4,6.7) & 5.1(1.6,7.5)\end{array}$

LupusQoL $^{\mathrm{e}}$-Physical Health (range 0-100, higher is better)

\begin{tabular}{|c|c|c|c|c|}
\hline Intervention & 25 & $\begin{array}{l}46.9(26.6, \\
56.3)\end{array}$ & $31.3(4.7,78.1)$ & $0.0(-18.8,29.7)$ \\
\hline Control & 22 & $\begin{array}{l}46.9(23.4, \\
60.9)\end{array}$ & $40.6(21.9,71.1)$ & $3.1(-10.2,10.2)$ \\
\hline
\end{tabular}

LupusQoL-Pain (range 0-100, higher is better)

$\begin{array}{lclll}\text { Intervention } & 25 & 41.7(20.8, & 41.7(0.0,83.3) & 8.3(-20.8,33.3) \\ & & 66.7) & \\ \text { Control } & 22 & \begin{array}{l}37.5(14.6, \\ 68.8)\end{array} & 33.3(14.6,66.7) & -4.2(-16.7,2.1)\end{array}$

LupusQoL-Planning (range 0-100, higher is better)

\begin{tabular}{|c|c|c|c|}
\hline Intervention & 25 & $41.7(8.3,70.8)$ & $50.0(0.0,91.7)$ \\
\hline Control & 22 & $\begin{array}{l}45.8(22.9, \\
75.0)\end{array}$ & $41.7(18.8,77.1)$ \\
\hline
\end{tabular}

LupusQoL-Burden to Others (range 0-100, higher is better)

$\begin{array}{llll}\text { Intervention } & 25 & 16.7(0.0,41.7) & 25.0(0.0,83.3) \\ \text { Control } & 22 & 20.8(0.0,45.8) & 41.7(0.0,77.1)\end{array}$

LupusQoL-Intimate Relationships (range 0-100, higher is better)

\begin{tabular}{|c|c|c|c|c|}
\hline Intervention & 19 & $\begin{array}{l}50.0(25.0, \\
75.0)\end{array}$ & $62.5(0.0,87.5)$ & $-12.5(-25.0,25.0)$ \\
\hline Control & 18 & $\begin{array}{l}56.3(25.0, \\
87.5)\end{array}$ & $62.5(25.0,100.0)$ & $0.0(-3.1,12.5)$ \\
\hline
\end{tabular}

LupusQoL-Emotional Health (range 0-100, higher is better)

$\begin{array}{lrlr}\text { Intervention } & 25 & 54.2(25.0, & 75.0(4.2,93.8) \\ & & 68.8) \\ \text { Control } & 22 & 52.1(29.2, & 56.3(37.5,70.8) \\ & & 70.8)\end{array}$

LupusQoL-Body Image (range 0-100, higher is better)

$\begin{array}{lrlrl}\text { Intervention } & 22 & \begin{array}{l}41.3(23.8, \\ 68.8)\end{array} & 51.9(0.0,90.0) & 13.1(-30.3,21.3) \\ \text { Control } & 19 & \begin{array}{l}35.0(20.0, \\ \text { 56.3) }\end{array} & 45.0(30.0,70.0) & 5.0(0.0,25.0)\end{array}$

$.04^{\mathrm{f}}$

.75

$-0.6$

.73

$0.0(-2.8,2.3)$

.76

.68

$-0.7$

.31

.16

.97

$\begin{array}{ll}-3.1 & .88\end{array}$

.64

.82

12.5

.21

.63

.28

0.0

$0.0(-12.5,25.0)$

.38

.36

$0.0(0.0,50.0)$

$8.3(0.0,16.7)$

$.02^{\mathrm{f}}$

$.04^{\mathrm{f}}$

$-12.5$

.79

.47

6.2

$8.3(-10.4,29.2)$

$2.1(-12.5,12.5)$

.30

.64

8.1

.76

$.047^{\mathrm{f}}$
.24

.92

.46

.37

.505

.88
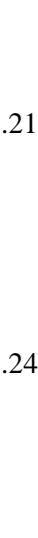

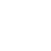

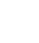

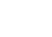

$9.4 \quad .22$ 


\begin{tabular}{|c|c|c|c|c|c|c|c|}
\hline \multirow[t]{2}{*}{ Domain } & \multicolumn{3}{|c|}{ Within group } & \multirow[b]{2}{*}{ Change in score (EOP: Baseline) } & \multicolumn{3}{|c|}{ Between group } \\
\hline & Count & Baseline & $\begin{array}{l}\text { End of program } \\
\text { (EOP) }\end{array}$ & & $P$-value & Difference $^{b}$ & $P$-value \\
\hline Intervention & 25 & $\begin{array}{l}25.0(15.6, \\
34.4)\end{array}$ & $62.5(0.0,81.3)$ & $18.8(-9.4,43.8)$ & $.007^{\mathrm{f}}$ & & \\
\hline Control & 22 & $25.0(4.7,39.1)$ & $31.3(15.6,53.1)$ & $9.4(-6.3,20.3)$ & $.03^{\mathrm{f}}$ & & \\
\hline
\end{tabular}

${ }^{\mathrm{a}}$ Within-group values are median (25th percentile, 75 th percentile). Nonparametric tests were chosen in order to require minimal distributional assumptions given the small sample size, and medians, 25th, and 75th percentile values are displayed as measures of central tendency and spread in order to be consistent with a nonparametric analysis. The within-group $P$-value is from the Wilcoxon signed-rank test and the between-group $P$-value is from the Mann-Whitney $U$ test; $P$-values themselves are unadjusted but the threshold for statistical significance is set using the Benjamini-Hochberg adjustment; LupusQoL-Intimate Relationships and LupusQoL-Body Image allow the possibility of N/A responses.

${ }^{\mathrm{b}}$ Difference in median change (intervention - control).

${ }^{c}$ FACIT-Fatigue: Functional Assessment of Chronic Illness Therapy-Fatigue (FACIT-F); 52-point scale with 0 (worst).

${ }^{\mathrm{d}}$ BPI-SF: Brief Pain Inventory-Short Form; 10-point scale with 0 (best).

${ }^{\mathrm{e}}$ LupusQoL: Lupus Quality of Life; 100-point scale with 0 (worst).

${ }^{\mathrm{f}}$ Statistically significant at an unadjusted 2-sided significance level of 5\%.

\section{Per-Protocol Analysis}

Within-group analysis of the PP intervention population revealed improvement over baseline at 16 weeks in all domains except LupusQoL-Intimate Relationships at unadjusted significance levels ( $P=.05$; Table 5). Adjusting for multiple comparisons, statistically significant improvement was achieved by the PP intervention group in 8 domains: FACIT-F (43.5 at the end of study vs 20.5 baseline; $P=.001$ ), LupusQoL-Fatigue ( 81.3 vs $28.1 ; P<.001)$, LupusQoL-Physical Health (71.9 vs 51.6; $P=.02)$, LupusQoL-Planning (83.3 vs 62.5; $P=.008)$, LupusQoL-Burden to Others (79.2 vs 25.0; $P=.003)$, LupusQoL-Emotional Health (83.3 vs $60.4 ; P=.01$ ), LupusQoL-Body Image ( 87.5 vs 56.3 , $P=.01$ ), and BPI-SF-Pain Interference ( 2.0 vs $6.3 ; P=.003)$. The usual care PP population had significant improvement over baseline for LupusQoL-Fatigue only (34.4 vs $25.0 ; P=.028)$.

Between-group comparisons demonstrated greater improvement in the intervention group than in the control group for every domain. Adjusting for multiple tests, significant differences in favor of the intervention group were reached in 6 domains: FACIT-F (difference in median changes of 18.0, $P<.001$ ), BPI-SF-Pain interference ( $-2.5, P=.02)$, LupusQoL-Pain (12.5, $P=.004)$ LupusQoL-Planning (16.7, $\quad P=.004)$, LupusQoL-Emotional Health (16.7, $P=.02)$, and LupusQoL-Fatigue $(25.0, P<.001)$. Three additional domains reached significance at an unadjusted level of 5\%: BPI-SF-Pain Severity ( $-1.9, P=.049)$, LupusQoL-Physical Health (14.1, $P=.049$ ), and LupusQoL-Burden to Others (29.2, $P=.04)$. The magnitude of the improvements in all domains (absolute and relative) is shown in Figure 3. Significantly greater improvement was seen in the intervention group compared with the control group. Results on an absolute basis are as follows: FACIT-F (34\% intervention vs $-1 \%$ control, $P<.001$ ), BPI-SF-Pain severity $(13 \%$ vs $-16 \%, P=.049)$, BPI-SF-Pain interference ( $25 \%$ vs $0 \%, P=.02$ ), and 4 LupusQoL measures, namely, pain (13\% vs $0 \%, P=.004)$, planning ( $17 \%$ vs $0 \%, P=.004)$, emotional health $(21 \%$ vs $4 \%, P=.02)$, and fatigue ( $38 \%$ vs $13 \%, P<.001)$. 
Table 5. Per-protocol analysis of change in FACIT, BPI-SF, and LupusQoL domain scores from baseline to end of program. ${ }^{\mathrm{a}}$

\begin{tabular}{|c|c|c|c|c|c|c|c|}
\hline \multirow[t]{2}{*}{ Domain } & \multicolumn{3}{|c|}{ Within group } & \multirow[b]{2}{*}{$\begin{array}{l}\text { Change in score } \\
\text { (EOP - Baseline) }\end{array}$} & \multirow[b]{2}{*}{$P$-value } & \multicolumn{2}{|c|}{ Between group } \\
\hline & Count & Baseline & $\begin{array}{l}\text { End of program } \\
\text { (EOP) }\end{array}$ & & & Difference $^{\mathrm{b}}$ & $P$-value \\
\hline \multicolumn{6}{|c|}{ FACIT $^{\mathrm{c}}$-Fatigue (range $0-52$, higher is better) } & 18.0 & $<.001^{\mathrm{f}}$ \\
\hline Intervention & 16 & $20.5(10.3,26.8)$ & $43.5(28.5,47.8)$ & $17.5(4.8,24.0)$ & $.001^{\mathrm{f}}$ & & \\
\hline Control & 18 & $20.5(14.0,27.3)$ & $22.0(12.5,28.3)$ & $-0.5(-4.3,7.3)$ & .79 & & \\
\hline \multicolumn{6}{|c|}{ BPI-SF $^{d}$-Pain Severity (range $0-10$, lower is better) } & -1.9 & $.049^{\mathrm{g}}$ \\
\hline Intervention & 16 & $4.8(3.0,6.5)$ & $3.3(1.6,5.2)$ & $-1.3(-3.0,0.4)$ & $.02^{\mathrm{g}}$ & & \\
\hline Control & 18 & $3.9(2.9,5.9)$ & $3.8(2.6,6.4)$ & $0.6(-1.3,1.0)$ & .68 & & \\
\hline \multicolumn{6}{|c|}{ BPI-SF-Pain Interference (range 0-10, lower is better) } & -2.5 & $.02^{\mathrm{f}}$ \\
\hline Intervention & 16 & $6.3(4.0,7.5)$ & $2.0(0.5,5.3)$ & $-2.5(-4.4,-0.2)$ & $.003^{\mathrm{f}}$ & & \\
\hline Control & 18 & $5.4(4.1,6.3)$ & $4.9(1.6,6.5)$ & $0.0(-1.2,0.8)$ & .64 & & \\
\hline \multicolumn{6}{|c|}{ LupusQoL $^{\mathrm{e}}$-Physical Health (range 0-100, higher is better) } & 14.1 & $.049^{\mathrm{g}}$ \\
\hline Intervention & 16 & $51.6(34.4,61.7)$ & $71.9(37.5,93.0)$ & $17.2(0.0,35.9)$ & $.02^{\mathrm{f}}$ & & \\
\hline Control & 18 & $51.6(27.3,65.6)$ & $48.4(26.6,71.1)$ & $3.1(-7.8,10.2)$ & .66 & & \\
\hline \multicolumn{6}{|c|}{ LupusQoL-Pain (range 0-100, higher is better) } & 12.5 & $.004^{\mathrm{f}}$ \\
\hline Intervention & 16 & $50.0(33.3,66.7)$ & $83.3(47.9,89.6)$ & $12.5(2.1,39.6)$ & $.03^{\mathrm{g}}$ & & \\
\hline Control & 18 & $58.3(22.9,75.0)$ & $41.7(16.7,66.7)$ & $0.0(-16.7,2.1)$ & .23 & & \\
\hline \multicolumn{6}{|c|}{ LupusQoL-Planning (range 0-100, higher is better) } & 16.7 & $.004^{\mathrm{f}}$ \\
\hline Intervention & 16 & $62.5(20.8,75.0)$ & $83.3(56.3,100.0)$ & $16.7(0.0,41.7)$ & $.008^{\mathrm{f}}$ & & \\
\hline Control & 18 & $62.5(25.0,75.0)$ & $41.7(25.0,77.1)$ & $0.0(-27.1,8.3)$ & .19 & & \\
\hline \multicolumn{6}{|c|}{ LupusQoL-Burden to Others (range 0-100, higher is better) } & 29.2 & $.04^{\mathrm{g}}$ \\
\hline Intervention & 16 & $25.0(2.1,54.2)$ & $79.2(31.3,83.3)$ & $33.3(0.0,58.3)$ & $.003^{\mathrm{f}}$ & & \\
\hline Control & 18 & $25.0(0.0,60.4)$ & $41.7(0.0,77.1)$ & $4.2(0.0,16.7)$ & .11 & & \\
\hline \multicolumn{6}{|c|}{ LupusQoL-Intimate Relationships (range 0-100, higher is better) } & 25.0 & .12 \\
\hline Intervention & 11 & $75.0(25.0,75.0)$ & $87.5(75.0,100.0)$ & $25.0(-12.5,50.0)$ & .06 & & \\
\hline Control & 15 & $62.5(25.0,87.5)$ & $50.0(25.0,87.5)$ & $0.0(-12.5,12.5)$ & .92 & & \\
\hline \multicolumn{6}{|c|}{ LupusQoL-Emotional Health (range 0-100, higher is better) } & 16.7 & $.02^{\mathrm{f}}$ \\
\hline Intervention & 16 & $60.4(34.4,81.3)$ & $83.3(68.8,99.0)$ & $20.8(4.2,37.5)$ & $.01^{\mathrm{f}}$ & & \\
\hline Control & 18 & $56.3(35.4,71.9)$ & $56.3(40.6,67.7)$ & $4.2(-9.4,12.5)$ & .57 & & \\
\hline \multicolumn{6}{|c|}{ LupusQoL-Body Image (range 0-100, higher is better) } & 13.8 & .09 \\
\hline Intervention & 13 & $56.3(14.4,69.4)$ & $87.5(68.8,95.0)$ & $18.8(13.1,46.9)$ & $.011^{\mathrm{f}}$ & & \\
\hline Control & 15 & $31.3(20.0,65.0)$ & $40.0(25.0,70.0)$ & $5.0(0.0,23.8)$ & .12 & & \\
\hline \multicolumn{6}{|c|}{ LupusQoL-Fatigue (range 0-100, higher is better) } & 25.0 & $<.001^{\mathrm{f}}$ \\
\hline Intervention & 16 & $28.1(25.0,53.1)$ & $81.3(64.1,92.2)$ & $37.5(21.9,48.4)$ & $<.001^{\mathrm{f}}$ & & \\
\hline Control & 18 & $25.0(10.9,43.8)$ & $34.4(25.0,53.1)$ & $12.5(-1.6,20.3)$ & $.03^{\mathrm{g}}$ & & \\
\hline
\end{tabular}

${ }^{\mathrm{a}}$ Within-group values are median (25th percentile, 75 th percentile). Nonparametric tests were chosen in order to require minimal distributional assumptions given the small sample size. Medians, 25th, and 75th percentile values are displayed as measures of central tendency and spread. The within-group $P$-value is from the Wilcoxon signed-rank test; the between-group $P$-value is from the Mann-Whitney $U$ test; $P$-values themselves are unadjusted but the threshold for statistical significance is set using the Benjamini-Hochberg adjustment. LupusQoL-Intimate Relationships and LupusQoL-Body Image allow N/A responses. 
${ }^{\mathrm{b}}$ Difference in median change (intervention - control).

${ }^{c}$ FACIT-Fatigue: Functional Assessment of Chronic Illness Therapy-Fatigue (FACIT-F); 52-point scale with 0 (worst).

${ }^{\mathrm{d}}$ BPI-SF: Brief Pain Inventory-Short Form; 10-point scale with 0 (best).

${ }^{\mathrm{e}}$ LupusQoL: Lupus Quality of Life; 100-point scale with 0 (worst).

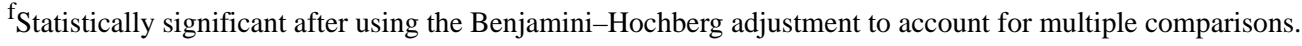

${ }^{\mathrm{g}}$ Statistically significant at an unadjusted 2-sided significance level of $5 \%$. 
Figure 3. Absolute and Relative Improvement by Domain. Absolute improvement was median change from baseline to endpoint divided by total possible domain score. Relative improvement was median change divided by the median baseline domain score. Changes in BPI-SF-pain interference and BPI-SF-pain severity are converted to positive $\%$ for consistency with other domains. $P$-values are from the Mann-Whitney $U$ test comparing changes in score between intervention and control groups. $P$-values are unadjusted. Although both ITT intervention and control groups achieved significant improvement in some domains, when the groups were compared, no statistically significant differences were found. ${ }^{\text {a }}$ Statistically significant after using the Benjamini-Hochberg adjustment ${ }^{b}$ Statistically significant at an unadjusted two-sided significance level of 5\%. FACIT: Functional Assessment of Chronic Illness Therapy; ITT: intention to treat.

\section{PP Absolute and Relative Improvement by Domain}

$$
\begin{array}{r}
\text { FACIT Fatigue }\left(P<.001^{\text {a }}\right) \\
\text { Pain Severity }\left(P=.05^{\mathrm{b}}\right) \\
\text { Pain Interference }\left(P=.02^{\mathrm{a}}\right) \\
\text { Physical Health }\left(P=.05^{\mathrm{b}}\right) \\
\text { Planning }\left(P=.004^{\mathrm{a}}\right) \\
\text { Pain }\left(P=.004^{\mathrm{a}}\right) \\
\text { Intimate Relationships }\left(P=.12^{2}\right) \\
\text { Emotional Health }\left(P=.02^{\mathrm{a}}\right) \\
\text { Body Image }(P=.09) \\
\text { Fatigue }\left(P<.001^{\text {a }}\right)
\end{array}
$$

\section{ITT Absolute and Relative Improvement by Domain}

$$
\begin{array}{r}
\text { FACIT Fatigue }(P=.12) \\
\text { Pain Severity }(P=.73) \\
\text { Pain Interference }(P=.31) \\
\text { Physical Health }(P=.88) \\
\text { Planning }(P=.24) \\
\text { Pain }(P=.21) \\
\text { Burden to Others }(P=.92) \\
\text { Intimate Relationships }(P=.46) \\
\text { Emotional Health }(P=.37) \\
\text { Body Image }(P=.51) \\
\text { Fatigue }(P=.22)
\end{array}
$$

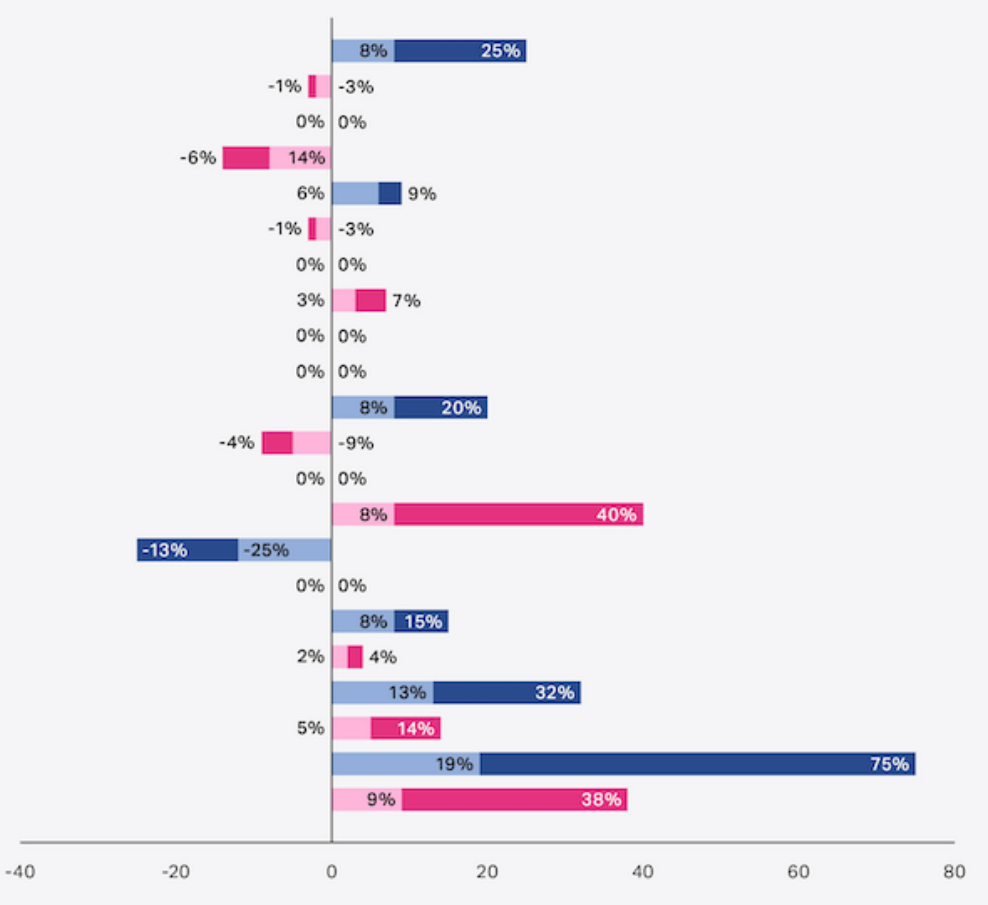

\section{Per-Protocol Analysis of Longitudinal Change}

Figures 4-7 depict change over time in FACIT-F, LupusQoL, and BPI-SF in both the ITT and PP groups. Improvement in the intervention group started in the first 4 weeks and continued through week 16 with the following exceptions: FACIT-F and LupusQoL-Fatigue had slightly higher improvement rates between weeks 4 and 8 and weeks 12 and 16 (Figure 4); a 
significant portion of pain reduction occurred between weeks 12 and 16 (Figure 7). The control group experienced modest improvement in LupusQoL-Fatigue at 12 weeks which diminished by week 16 , whereas all other domains remained largely unchanged or deteriorated over the course of the 16 weeks.

Figure 4. Change over time in FACIT-Fatigue and LupusQoL-Fatigue. FACIT: Functional Assessment of Chronic Illness Therapy; LupusQoL: Lupus Quality of Life.

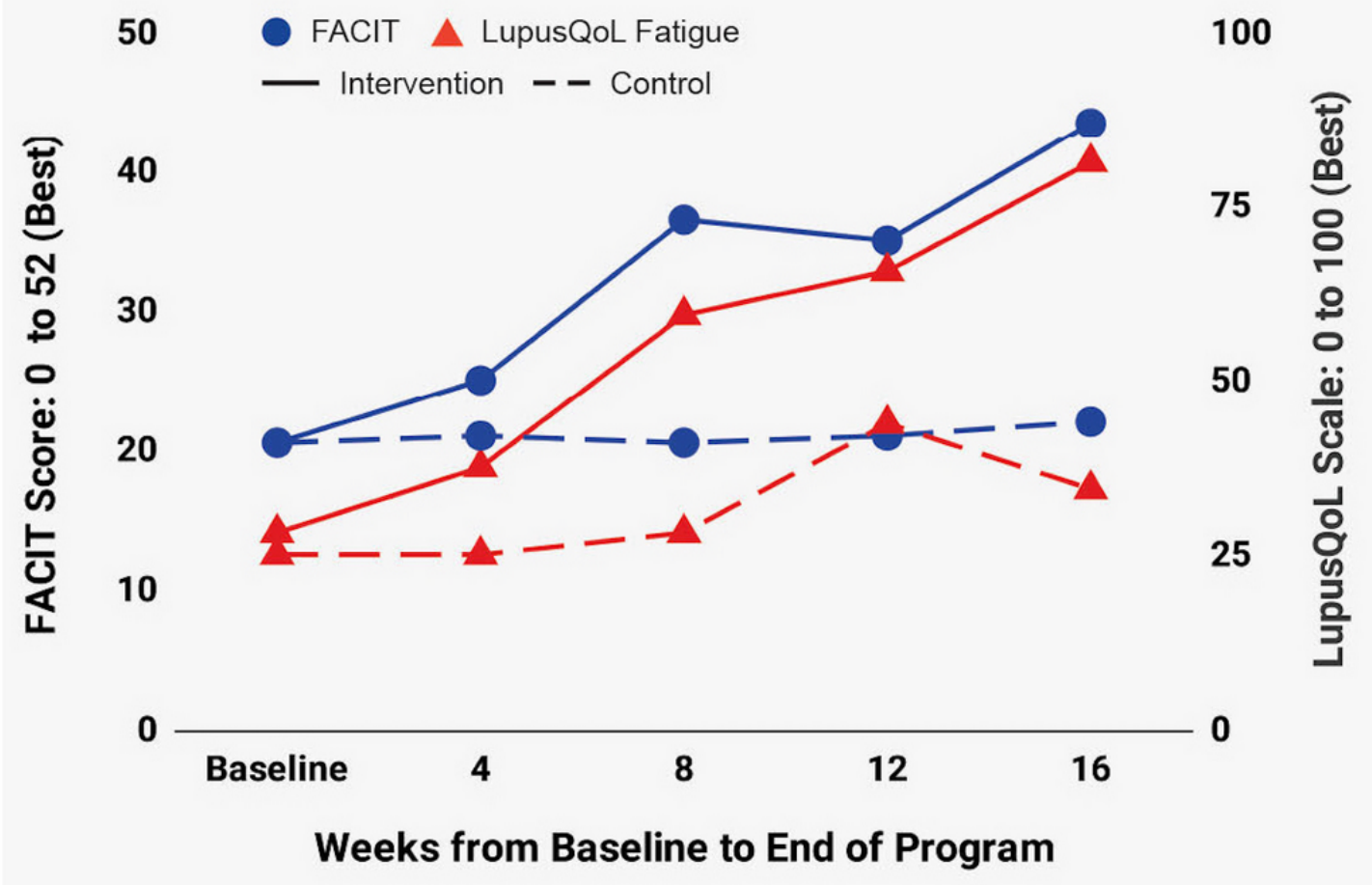

Figure 5. Change over time in LupusQoL-Physical Health, LupusQoL-Pain and LupusQoL-Burden to Others. LupusQoL: Lupus Quality of Life.

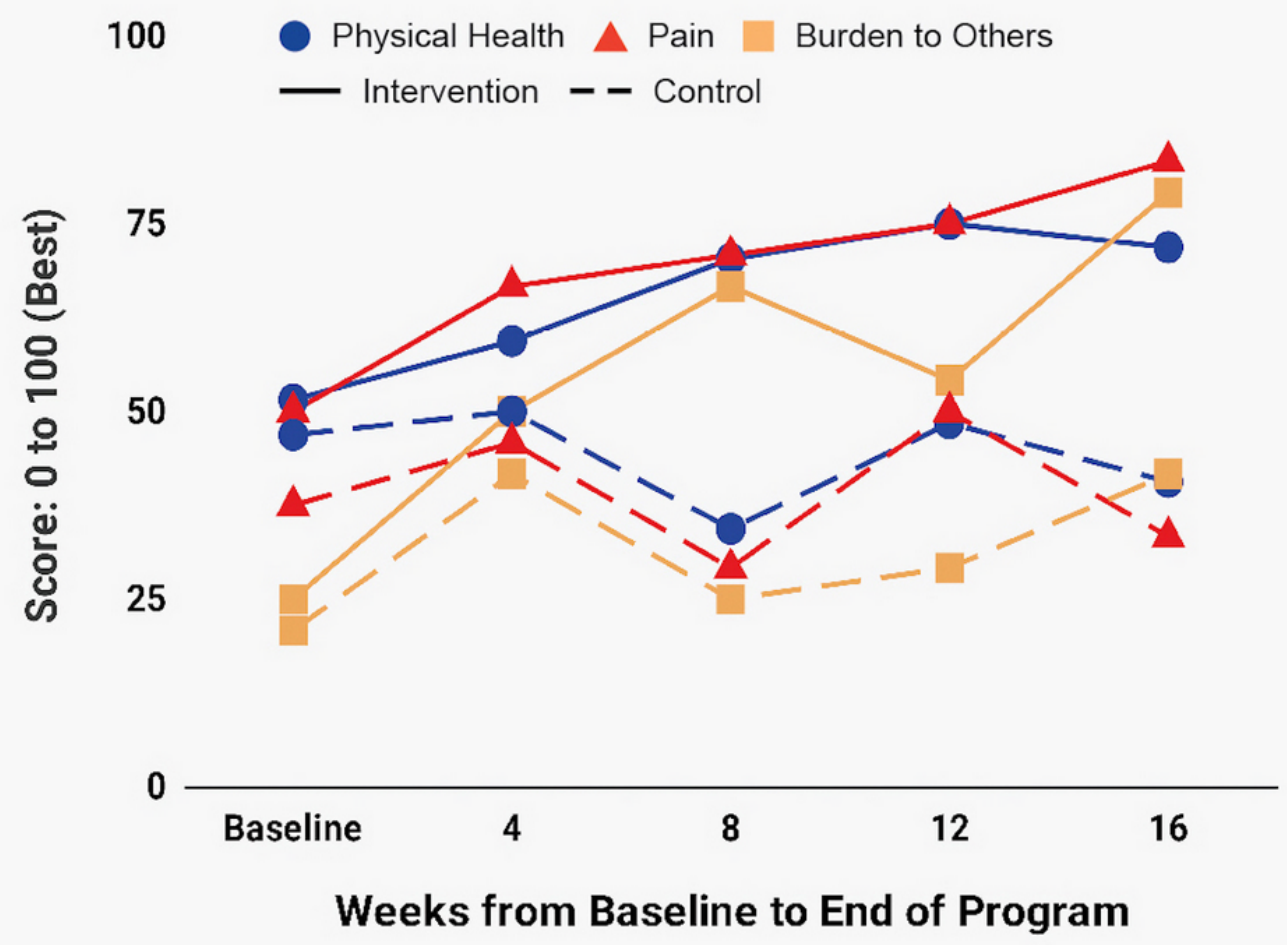


Figure 6. Change over time in LupusQoL-Planning, LupusQoL-Relationships, LupusQoL-Emotional Health and LupusQoL-Body Image. LupusQoL: Lupus Quality of Life.

100

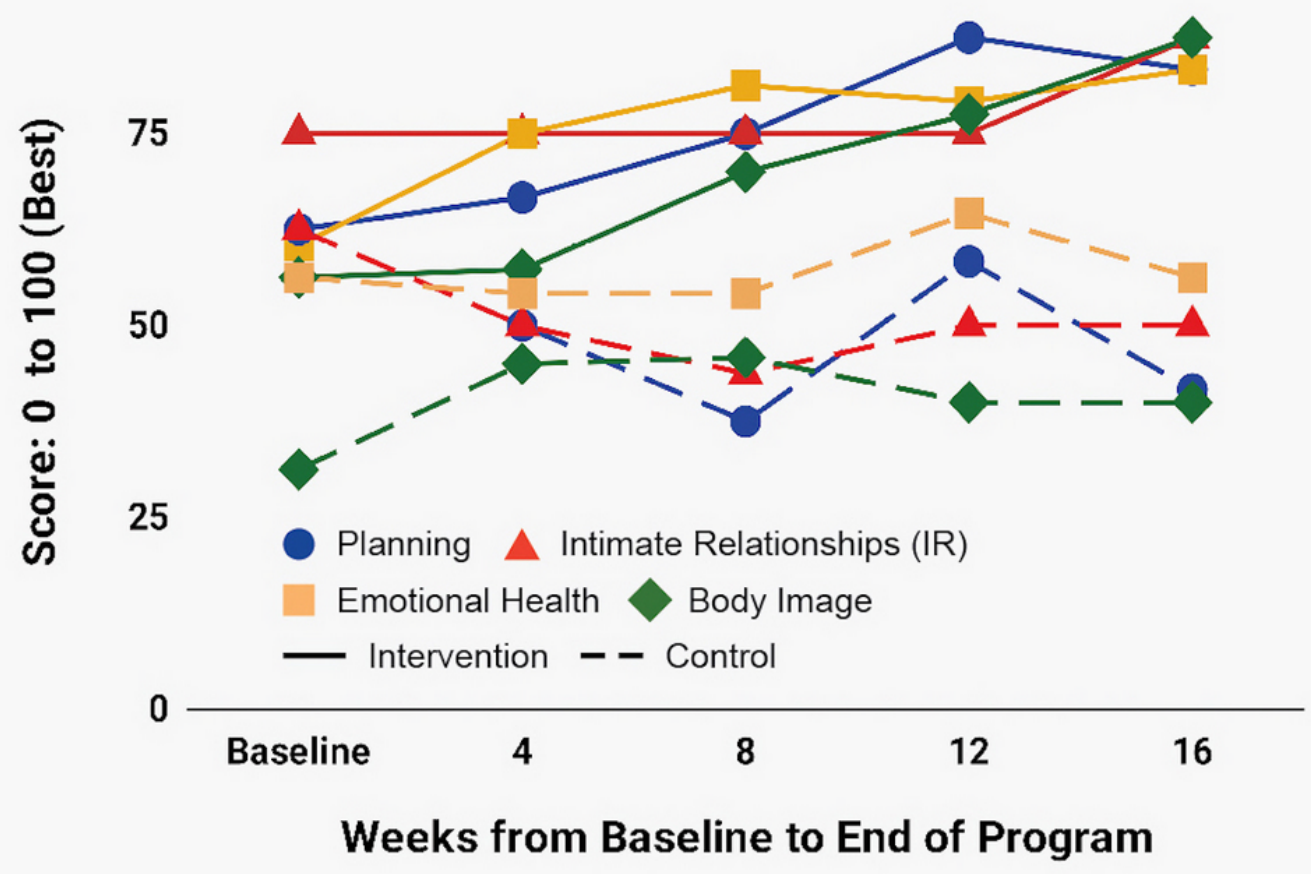

Figure 7. Change over time in BPI-SF Pain Severity and BPI-SF Pain Interference. BPI-SF: Brief Pain Index-Short Form.

8

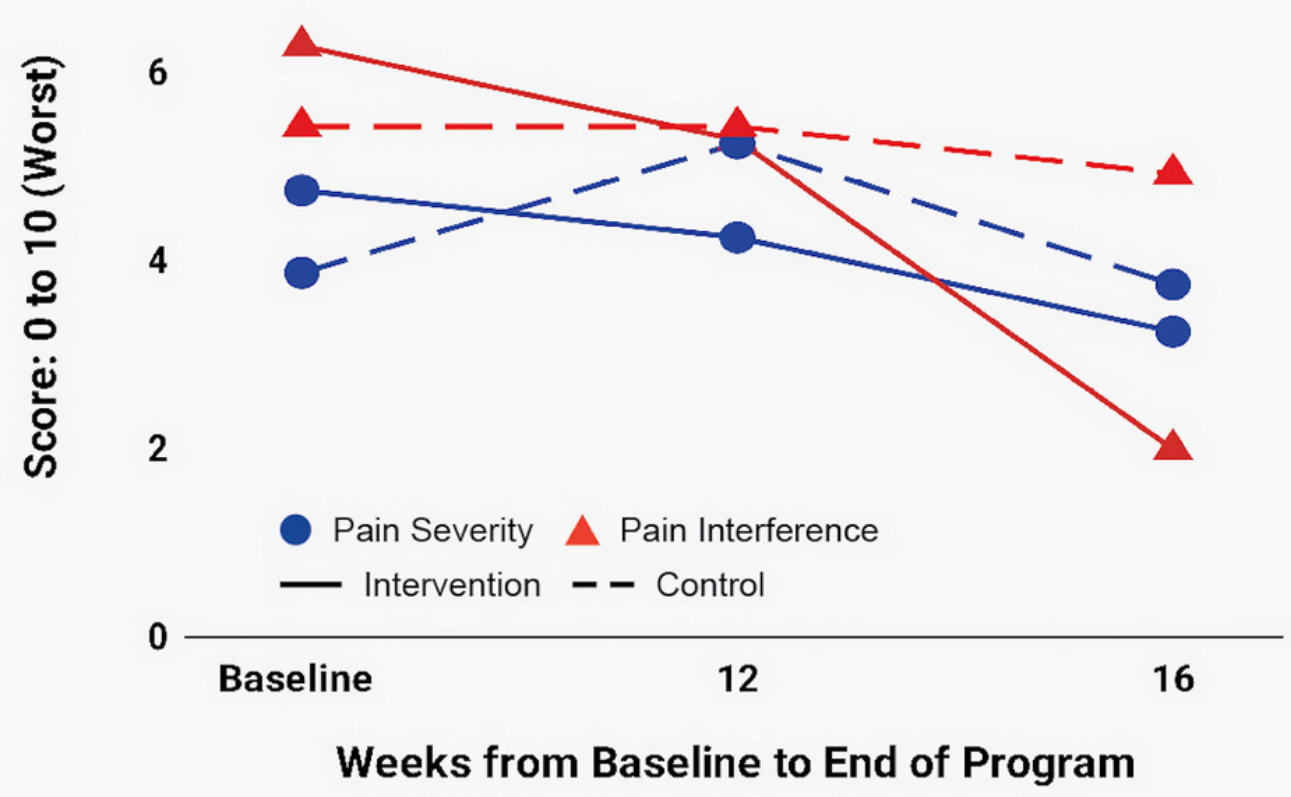

\section{Frequency of Tracked Symptoms, Triggers, and Interventions}

Table 6 displays the top 3 participant-tracked inputs (symptoms and suspected triggers) and top 4 coach-recommended interventions in the ITT and PP groups. Among both groups, the 3 most frequently tracked symptoms were fatigue, joint pain, and brain fog. The most common triggers in both groups were dairy, gluten, and nightshades (a family of plants that include potatoes, tomatoes, capsicum, bell peppers, eggplant, and tobacco). The most commonly recommended interventions, aside from ensuring adequate hydration, were dietary elimination 
of triggers and the addition of digestive enzyme supplements, apple cider vinegar, and protein shakes.

Table 6. Frequency of tracked symptoms, triggers, and interventions. ${ }^{\text {a }}$

\begin{tabular}{|c|c|c|c|c|}
\hline \multirow[t]{2}{*}{ Variable } & \multicolumn{2}{|c|}{ Intention to treat } & \multicolumn{2}{|c|}{ Per protocol } \\
\hline & $\mathrm{n}$ & Relative frequency (\%) & $\mathrm{n}$ & Relative frequency (\%) \\
\hline \multicolumn{5}{|l|}{ Interventions } \\
\hline Dietary elimination & 17 & 68 & 16 & 100 \\
\hline Digestive enzymes & 19 & 76 & 16 & 100 \\
\hline Protein shake & 17 & 68 & 15 & 94 \\
\hline Apple cider vinegar & 15 & 60 & 14 & 88 \\
\hline \multicolumn{5}{|l|}{ Triggers } \\
\hline Dairy & 19 & 76 & 14 & 88 \\
\hline Gluten & 14 & 56 & 11 & 69 \\
\hline Nightshades & 7 & 28 & 6 & 38 \\
\hline \multicolumn{5}{|l|}{ Symptoms } \\
\hline Joint pain & 6 & 24 & 6 & 38 \\
\hline Brain fog & 6 & 24 & 6 & 38 \\
\hline Fatigue & 7 & 28 & 5 & 31 \\
\hline
\end{tabular}

${ }^{\mathrm{a}}$ Total population sampled includes intention to treat $(\mathrm{N}=25)$ and per protocol $(\mathrm{N}=16)$.

\section{Adverse Events}

No adverse events attributable to the intervention occurred. Four participants ( 3 from the control group and 1 from the intervention group) experienced SLE exacerbations requiring pulse steroids within the last 4 weeks of the study.

\section{Discussion}

\section{Principal Findings}

To the authors' knowledge, this is the first study to show that a digital therapeutic intervention targeting dietary, environmental, and lifestyle factors can improve HRQoL when added to usual care in patients with SLE. Participants who completed the 16-week protocol showed continuous improvement across all HRQoL domains and statistically greater improvement than those receiving usual care alone for the majority of domains. Of particular interest is the significant improvement noted in fatigue. Fatigue is one of the most debilitating symptoms reported by patients with SLE; is highly correlated with work disability [38], workplace absence (absenteeism), or impaired workplace performance (presenteeism) [39,40]; and is frustratingly recalcitrant to treatment. Although not formally assessed (and therefore only serves as a point for further exploration in future studies), qualitative data collected via coaching notes revealed that 2 participants in the PP intervention group who had been on disability at the start of the study (15 years and 3 years) felt ready for work.

In this SLE population, the most common triggers identified as correlating negatively with symptoms and leading to improvement upon elimination were all dietary - the top 3 being dairy, gluten, and nightshades. While these findings do not provide conclusive evidence linking dairy, gluten, and nightshades to SLE, accumulating data from in vitro, animal, and human studies support the need for ongoing investigation into these potential triggers [41-43].

Elimination of food triggers identified by the program's software as aggravating symptoms was central to the therapeutic approach of this platform. In addition, a variety of low-risk, nutritional interventions not previously studied in an SLE population were frequently incorporated into the participants' personalized plans. These interventions were primarily aimed at improving the participants' numerous digestive and energy-level complaints which weigh heavily on HRQoL in SLE and included digestive enzymes, small amounts of apple cider vinegar, and protein shakes.

Progress in the care of patients with SLE has been slow, largely attributable to inherent disease characteristics as well as health care access and socioeconomic obstacles. As discussed, disease heterogeneity is perhaps the most significant obstacle to progress. Other barriers to advancement are lack of diagnostic, predictive, prognostic, and drug-response biomarkers; ineffective management of SLE due to social determinants of care in predominantly lower socioeconomic status areas; and lack of treatment adherence [24]. Nontraditional solutions to these challenges should be explored and digital therapeutics offer one such novel approach. The digital therapeutic intervention tested here focused on identifying and eliminating dietary, environmental, and lifestyle triggers of SLE as an adjunct to usual care. This approach implicitly takes disease heterogeneity into account, leverages the growing understanding of the role environment plays in initiating and propagating SLE, and personalizes each patient's recommendations supported by software data analytics. This personalized approach is especially 
intriguing as it applies to dietary interventions in SLE. A recent review article assessing the significance and impact of dietary factors on SLE pathogenesis found that small and personalized improvements in diet could alter the clinical status of patients with SLE and concluded that "proper diet in SLE can help preserve the body's homeostasis, increase the period of remission, prevent adverse effects of medication [especially systemic corticotherapy] and improve the patient's physical and mental well-being" [44].

With on-going research, digital therapeutics may hold the key to overcoming many barriers to SLE care. The enormity of data that can be collected and analyzed via a digital therapeutic platform has the potential to help identify new SLE biomarkers. Aspects of care that prove difficult to deliver to disadvantaged populations with the traditional medical model may be made more accessible. Importantly, if larger studies validate these preliminary findings and build on this work by demonstrating improvements in disease activity measures (eg, Systemic Lupus Erythematosus Disease Activity Index [SLEDAI], SLE Responder Index [SRI]), then dietary and lifestyle interventions delivered in conjunction with a digital therapeutic device may allow for more selective and conservative use of costly, potentially dangerous immune-modulating drugs. Medication changes were not formally followed in this study but information from coaching notes revealed that 5 study participants in the PP intervention group were able to reduce or discontinue immune-modulating medications. In addition, several participants reduced or discontinued use of multiple symptom-relieving medications (including over-the-counter and prescription drugs for gastrointestinal symptoms, pain, depression, and anxiety). As medication usage was not a prespecified outcome in this trial and was not formally assessed, conclusions about the impact of this intervention on medication usage cannot be made. However, if these results are reproduced in larger studies (in which medication information is formally collected and analyzed) the implications of drug reduction alone are important. Polypharmacy is highly prevalent in SLE [45], is associated with elevated risk of adverse drug events, and was shown in a 2017 meta-analysis to be linked to increased mortality [46].

It was not possible in this small pilot trial to examine the underlying physiological mechanisms responsible for patients' improvements. Provocative findings from several lines of research compel one to consider the effects that the collection of lifestyle modifications, particularly dietary changes, may have had on the health of the intestinal epithelium and the gut microbiome. In animal [14,47-49] and human [50,51] studies, mounting evidence points to a central role of the intestinal epithelial barrier and related diversity and function of the gut microbiome in autoimmune disease. In 2014, the National Institutes of Health (NIH) launched the Integrative Human Microbiome Project "to generate resources to permit comprehensive characterization of the human microbiota to further our understanding of how the microbiome impacts human health and disease" [51]. One of the 3 microbiome-associated conditions which are being explored is autoimmune in nature, namely, inflammatory bowel disease. As microbiome characteristics have also been implicated in
SLE, it would be valuable in future studies of this digital therapeutic to assess microbiome composition before and after the intervention.

This exploratory pilot study has many limitations and the results should be interpreted in this context. Physician-scored, validated SLE disease activity measures (such as British Isles Lupus Assessment Group or SLEDAI) were not captured, limiting the capacity to assess disease severity at baseline and change in disease activity by strict clinical criteria throughout the study. In this exploratory pilot study, limited budget and research manpower restricted the ability to pursue this depth of data collection. While inclusion of such clinical disease activity scores in future, larger studies is planned and will provide critical insights, absence of these measures should not diminish the relevance of HRQoL outcomes. The debilitating symptoms, toxicity of immune-modulating treatments, unpredictability of disease activity, and fear of serious, even life-threatening manifestations associated with SLE have a profound impact on HRQoL across multiple domains. These features are not adequately captured by clinical measures of disease activity, which previous research has shown to have poor correlation with patient assessment of HRQoL [52]. Furthermore, HRQoL has been found to be associated with treatment adherence and health care utilization in patients with SLE [53]. The PROMs utilized in this study were chosen to capture many of the diverse domains that contribute to the complex concept of HRQoL.

Selection bias may have been introduced by heavy recruitment from online SLE and other autoimmune patient websites and therefore the study group may not be representative of the general SLE population. However, the number of patients who seek online medical advice is large; continues to grow; and crosses gender, age, and socioeconomic differences [54]. Selection bias may have also been introduced by the requirement of owning a smartphone. Smartphone ownership, however, has become increasingly common across gender, race, education, and economic levels [55], hopefully minimizing this bias. But, in future studies this can be addressed by providing smartphones for those in need.

This study failed to show statistically significant between-group differences in any measured domain in the ITT analysis. These results were affected by disproportionate attrition from the intervention group early in the study (Figure 2). Six intervention participants $(6 / 25,24 \%)$ left the study after having completed 0 to 1 of 16 sessions, whereas only 1 control group participant was lost early. Missing data from participants who dropped out of the intervention group were populated with the worst observed scores for that time point, thus biasing toward the null hypothesis. Furthermore, while 1 participant in the intervention group did receive pulse steroids within the last 4 weeks of the study period, excluding her from PP analysis, 3 patients in the control group also received pulse steroids in this time frame. Any positive effect this treatment had on outcomes would have biased toward the null hypothesis. The attrition rate may speak to the requirements inherent in this type of intervention, namely, that participants need to be motivated and engaged with an aptitude for regular app use and an interest in attending weekly coaching sessions. In future studies, early attrition will be addressed by building a run-in period into the design to help 
mitigate this issue. That this intervention has shown an $83 \%$ completion rate (participating in at least twelve of sixteen coaching sessions) in 70 autoimmune patients from a private insurance cohort is reassuring that the program has acceptable usability (internal data).

As potential adverse events were collected for the control group only at the end of the study (as opposed to the intervention group who were queried about potential adverse events on weekly coaching calls), these data may have been subject to recall bias. Future studies, which are planned to include a sham app and weekly sham coaching calls (see below), will overcome this potential bias.

There was no sham app or sham coaching in this study. Digital apps and health coaching alike are intrinsically engaging, thus vulnerable to the placebo response. It is not possible to tell to what extent HRQoL improvements were influenced by this engagement and patient expectations rather than the program interventions. Future studies should include a convincing sham app and interaction between controls and a health coach at the same frequency as that which occurs with the intervention group. Development of a sham app and sham coaching protocols are underway.

\section{Conclusions}

In conclusion, the digital therapeutic and coaching intervention tested in this pilot trial resulted in statistically significant, clinically meaningful improvements when added to usual care, compared with usual care alone, in several measures of HRQoL (including pain and fatigue) in adult patients with SLE. The study demonstrated that an adaptive, multifaceted intervention which aims at identifying and limiting each SLE participant's specific dietary and environmental triggers can improve symptoms and HRQoL without additional pharmaceutical manipulation of the immune system. These promising results stimulate a call for a larger study that includes measurement of validated SLE disease activity measures, sham controls, analysis of the biological mechanisms that underlie the improvements, and long-term follow-up of patients to confirm sustained gains in HRQoL. Broad adoption of the intervention could assist in building a database of SLE triggers which could deepen the understanding of the etiology of this disease and potentially contribute to SLE prevention in the future. Finally, given the expected role of diet, environment, and lifestyle in other autoimmune diseases, many of which have gaps in care similar to those in SLE, studies of the intervention's application to other autoimmune conditions is warranted.

\title{
Acknowledgments
}

We express our deepest gratitude to Vanessa Rudin for managing the research team and study deliverables and to Dr Vincent Campasano, who oversaw the safety of the trial. We extend our appreciation to Mette Dyhrberg (Founder, Mymee, Inc; Certified Integrative Health and Wellness Coach) for her compassionate and skilled health coaching services. We also thank Charlotta Norgaard (Lupus Friends and Family) for promoting the study to potential participants and providing feedback from her Facebook group members about the recruiting process and materials; Niecy Rivera (Flare Fighters), Aaron Jun and Lily Stairs (Clara Health), Arif Sorathia and Brett Adelman (LupusCorner), Aaron Abend (Autoimmune Registry), and Skylar Schone (MyID) for promoting the study to potential participants. And we thank the participants for having the courage to try something different. This study is supported by funds from the sponsor, Mymee, Inc.

\section{Authors' Contributions}

All co-authors contributed substantially to the analysis and interpretation of the data and provided important intellectual input. FK and NG wrote the first draft of the manuscript with editing of analysis, content, and format by YC and RM. All authors and the statistician agreed that the accuracy and integrity of the work has been appropriately investigated and resolved, and all approved the final version of the manuscript. The corresponding author had full access to the data and had final responsibility for the decision to submit for publication. The corresponding author attests that all listed authors meet authorship criteria and that no others meeting the criteria have been omitted.

\section{Conflicts of Interest}

FK and RM have received standard financial stipends from Mymee, Inc. and have no other disclosures to make. NG and YC have nothing to declare.

\section{Multimedia Appendix 1}

Supplement Protocol.

[DOCX File, 17 KB-Multimedia Appendix 1]

\author{
Multimedia Appendix 2 \\ CONSORT EHEALTH checklist (v 1.6.2). \\ [PDF File (Adobe PDF File), $101 \mathrm{~KB}-$ Multimedia Appendix 2]
}

\section{References}


1. Lupus Facts and Statistics. 2016 Oct 06. URL: https://www.lupus.org/resources/ lupus-facts-and-statistics\#: :text=The $\% 20$ Lupus $\% 20$ Foundation $\% 20$ of $\% 20$ America,mostly $\% 20$ women $\% 20$ of $\% 20$ childbearing $\% 20$ age [accessed 2020-08-03]

2. Lightstone L, Doria A, Wilson H, Ward FL, Larosa M, Bargman JM. Can we manage lupus nephritis without chronic corticosteroids administration? Autoimmun Rev 2018 Jan;17(1):4-10. [doi: 10.1016/j.autrev.2017.11.002] [Medline: 29108828]

3. Pons-Estel GJ, Alarcón GS, Scofield L, Reinlib L, Cooper GS. Understanding the epidemiology and progression of systemic lupus erythematosus. Semin Arthritis Rheum 2010 Feb;39(4):257-268 [FREE Full text] [doi: 10.1016/j.semarthrit.2008.10.007] [Medline: 19136143 ]

4. Bernatsky S, Boivin J, Joseph L, Manzi S, Ginzler E, Gladman DD, et al. Mortality in systemic lupus erythematosus. Arthritis Rheum 2006 Aug;54(8):2550-2557 [FREE Full text] [doi: 10.1002/art.21955] [Medline: 16868977]

5. Yen EY, Shaheen M, Woo JMP, Mercer N, Li N, McCurdy DK, et al. 46-Year Trends in Systemic Lupus Erythematosus Mortality in the United States, 1968 to 2013: A Nationwide Population-Based Study. Ann Intern Med 2017 Dec 05;167(11):777-785 [FREE Full text] [doi: 10.7326/M17-0102] [Medline: 29086801]

6. Almehed K, Carlsten H, Forsblad-d'Elia H. Health-related quality of life in systemic lupus erythematosus and its association with disease and work disability. Scand J Rheumatol 2010;39(1):58-62. [doi: 10.3109/03009740903124408] [Medline: 20132072]

7. Olesińska M, Saletra A. Quality of life in systemic lupus erythematosus and its measurement. Reumatologia 2018;56(1):45-54 [FREE Full text] [doi: 10.5114/reum.2018.74750] [Medline: 29686443]

8. Utset TO, Chohan S, Booth SA, Laughlin JC, Kocherginsky M, Schmitz A. Correlates of formal work disability in an urban university systemic lupus erythematosus practice. J Rheumatol 2008 Jun;35(6):1046-1052. [Medline: 18464309]

9. Yen EY, Singh RR. Brief Report: Lupus-An Unrecognized Leading Cause of Death in Young Females: A Population-Based Study Using Nationwide Death Certificates, 2000-2015. Arthritis Rheumatol 2018 Aug;70(8):1251-1255 [FREE Full text] [doi: 10.1002/art.40512] [Medline: 29671279]

10. Mazzone R, Zwergel C, Artico M, Taurone S, Ralli M, Greco A, et al. The emerging role of epigenetics in human autoimmune disorders. Clin Epigenetics 2019 Feb 26;11(1):34 [ [FREE Full text] [doi: 10.1186/s13148-019-0632-2] [Medline: 30808407]

11. Riscuta G, Xi D, Pierre-Victor D, Starke-Reed P, Khalsa J, Duffy L. Diet, Microbiome, and Epigenetics in the Era of Precision Medicine. Methods Mol Biol 2018;1856:141-156. [doi: 10.1007/978-1-4939-8751-1_8] [Medline: 30178250]

12. Pan Y, Ke H, Yan Z, Geng Y, Asner N, Palani S, et al. The western-type diet induces anti-HMGB1 autoimmunity in Apoe(-/-) mice. Atherosclerosis 2016 Aug;251:31-38 [FRE Full text] [doi: 10.1016/j.atherosclerosis.2016.05.027] [Medline: 27240253]

13. Mu Q, Zhang H, Luo XM. SLE: Another Autoimmune Disorder Influenced by Microbes and Diet? Front Immunol 2015;6:608 [FREE Full text] [doi: 10.3389/fimmu.2015.00608] [Medline: 26648937]

14. Parks CG, de Souza Espindola Santos A, Barbhaiya M, Costenbader KH. Understanding the role of environmental factors in the development of systemic lupus erythematosus. Best Pract Res Clin Rheumatol 2017 Jun;31(3):306-320 [FREE Full text] [doi: 10.1016/j.berh.2017.09.005] [Medline: 29224673]

15. Hardy TM, Tollefsbol TO. Epigenetic diet: impact on the epigenome and cancer. Epigenomics 2011 Aug;3(4):503-518 [FREE Full text] [doi: 10.2217/epi.11.71] [Medline: 22022340]

16. Young KA, Munroe ME, Harley JB, Guthridge JM, Kamen DL, Gilkensen GS, et al. Less than 7 hours of sleep per night is associated with transitioning to systemic lupus erythematosus. Lupus 2018 Aug;27(9):1524-1531 [FREE Full text] [doi: 10.1177/0961203318778368] [Medline: 29804502]

17. Gaine ME, Chatterjee S, Abel T. Sleep Deprivation and the Epigenome. Front Neural Circuits 2018;12:14 [FREE Full text] [doi: 10.3389/fncir.2018.00014] [Medline: 29535611]

18. Hsiao Y, Chen Y, Tseng C, Wu L, Lin W, Su VY, et al. Sleep disorders and increased risk of autoimmune diseases in individuals without sleep apnea. Sleep 2015 Apr 01;38(4):581-586 [FREE Full text] [doi: 10.5665/sleep.4574] [Medline: 25669189]

19. Song H, Fang F, Tomasson G, Arnberg FK, Mataix-Cols D, Fernández de la Cruz L, et al. Association of Stress-Related Disorders With Subsequent Autoimmune Disease. JAMA 2018 Jun 19;319(23):2388-2400 [FREE Full text] [doi: 10.1001/jama.2018.7028] [Medline: 29922828]

20. Gudsnuk K, Champagne FA. Epigenetic influence of stress and the social environment. ILAR J 2012;53(3-4):279-288 [FREE Full text] [doi: 10.1093/ilar.53.3-4.279] [Medline: 23744967]

21. Lee RS, Tamashiro KLK, Yang X, Purcell RH, Harvey A, Willour VL, et al. Chronic corticosterone exposure increases expression and decreases deoxyribonucleic acid methylation of Fkbp5 in mice. Endocrinology 2010 Sep;151(9):4332-4343 [FREE Full text] [doi: 10.1210/en.2010-0225] [Medline: 20668026]

22. Simpson R, Kunz H, Agha N, Graff R. Exercise and the Regulation of Immune Functions. Prog Mol Biol Transl Sci 2015;135:355-380. [doi: 10.1016/bs.pmbts.2015.08.001] [Medline: 26477922]

23. Zhang FF, Cardarelli R, Carroll J, Zhang S, Fulda KG, Gonzalez K, et al. Physical activity and global genomic DNA methylation in a cancer-free population. Epigenetics 2011 Mar;6(3):293-299 [FREE Full text] [doi: 10.4161/epi.6.3.14378] [Medline: 21178401] 
24. Manzi S, Raymond S, Tse K, Peña Y, Anderson A, Arntsen K, et al. Global consensus building and prioritisation of fundamental lupus challenges: the ALPHA project. Lupus Sci Med 2019;6(1):e000342. [doi: 10.1136/lupus-2019-000342] [Medline: 31413854]

25. Examples of Device Software Functions the FDA Regulates. Silver Spring, MD: Food and Drug Administration; 2019 Sep 26. URL: https://www.fda.gov/medical-devices/device-software-functions-including-mobile-medical-applications/ examples-device-software-functions-fda-regulates [accessed 2020-08-02]

26. Coravos A, Khozin S, Mandl KD. Developing and adopting safe and effective digital biomarkers to improve patient outcomes. NPJ Digit Med 2019;2(1):40 [FREE Full text] [doi: 10.1038/s41746-019-0090-4] [Medline: 30868107]

27. Gordon WJ, Landman A, Zhang H, Bates DW. Beyond validation: getting health apps into clinical practice. NPJ Digit Med 2020;3:14 [FREE Full text] [doi: 10.1038/s41746-019-0212-z] [Medline: 32047860]

28. Us in Lupus. URL: https://www.usinlupus.com/tools/mylupuslog.html [accessed 2020-08-02]

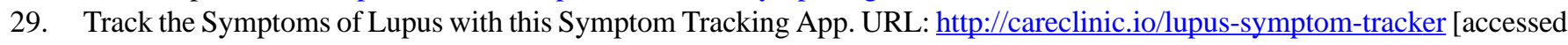
2020-08-02]

30. My Lupus Log. URL: http://myhealthapps.net/app/details/410/my-lupus-log

31. Mango Health. URL: https://www.mangohealth.com/ [accessed 2020-09-18]

32. McElhone K, Abbott J, Shelmerdine J, Bruce IN, Ahmad Y, Gordon C, et al. Development and validation of a disease-specific health-related quality of life measure, the LupusQol, for adults with systemic lupus erythematosus. Arthritis Rheum 2007 Aug 15;57(6):972-979 [FREE Full text] [doi: 10.1002/art.22881] [Medline: 17665467]

33. Lai J, Beaumont JL, Ogale S, Brunetta P, Cella D. Validation of the functional assessment of chronic illness therapy-fatigue scale in patients with moderately to severely active systemic lupus erythematosus, participating in a clinical trial. J Rheumatol 2011 Apr;38(4):672-679. [doi: 10.3899/jrheum.100799] [Medline: 21239746]

34. Mease PJ, Spaeth M, Clauw DJ, Arnold LM, Bradley LA, Russell IJ, et al. Estimation of minimum clinically important difference for pain in fibromyalgia. Arthritis Care Res (Hoboken) 2011 Jun;63(6):821-826 [FREE Full text] [doi: 10.1002/acr.20449] [Medline: 21312349]

35. Izadi Z, Gandrup J, Katz PP, Yazdany J. Patient-reported outcome measures for use in clinical trials of SLE: a review. Lupus Sci Med 2018;5(1):e000279. [doi: 10.1136/lupus-2018-000279] [Medline: 30167315]

36. Naegeli AN, Tomaszewski EL, Al Sawah S. Psychometric validation of the Brief Pain Inventory-Short Form in patients with systemic lupus erythematosus in the United States. Lupus 2015 Nov;24(13):1377-1383. [doi:

10.1177/0961203315588972] [Medline: 26038345]

37. McElhone K, Abbott J, Sutton C, Mullen M, Lanyon P, Rahman A, et al. Sensitivity to Change and Minimal Important Differences of the LupusQoL in Patients With Systemic Lupus Erythematosus. Arthritis Care Res (Hoboken) 2016 Oct;68(10):1505-1513 [FREE Full text] [doi: 10.1002/acr.22850] [Medline: 26816223]

38. Allaire S. Is work disability associated with systemic lupus erythematosus modifiable? J Rheumatol 2008 Jun;35(6):953-955. [Medline: 18528948]

39. Scofield L, Reinlib L, Alarcón GS, Cooper GS. Employment and disability issues in systemic lupus erythematosus: a review. Arthritis Rheum 2008 Oct 15;59(10):1475-1479 [FREE Full text] [doi: 10.1002/art.24113] [Medline: 18821664 ]

40. Connolly D, Fitzpatrick C, O'Toole L, Doran M, O'Shea F. Impact of Fatigue in Rheumatic Diseases in the Work Environment: A Qualitative Study. Int J Environ Res Public Health 2015 Oct 28;12(11):13807-13822 [FREE Full text] [doi: 10.3390/ijerph121113807] [Medline: 26516896]

41. Arroyo Hornero R, Hamad I, Côrte-Real B, Kleinewietfeld M. The Impact of Dietary Components on Regulatory T Cells and Disease. Front Immunol 2020;11:253 [FREE Full text] [doi: 10.3389/fimmu.2020.00253] [Medline: 32153577]

42. Hussain N. N282: Effect of gluten containing diet on pristane induced lupus prone mice. Lupus Science and Medicine 2017;4(Suppl_1):A129-A130 [FREE Full text] [doi: 10.1136/lupus-2017-000215.282]

43. Coucke F. Food intolerance in patients with manifest autoimmunity. Observational study. Autoimmun Rev 2018 Nov;17(11):1078-1080. [doi: 10.1016/j.autrev.2018.05.011] [Medline: 30213697]

44. Constantin M, Nita IE, Olteanu R, Constantin T, Bucur S, Matei C, et al. Significance and impact of dietary factors on systemic lupus erythematosus pathogenesis. Exp Ther Med 2019 Feb;17(2):1085-1090 [FREE Full text] [doi: 10.3892/etm.2018.6986] [Medline: 30679978]

45. Seguin D, Peschken C, Dolovich C, Grymonpre R, St JP, Tisseverasinghe A. Polypharmacy and potentially inappropriate medication use in young vs older adults with SLE (abstract). 2019 Presented at: 2019 ACR/ARP Annual Meeting; November 8-13, 2019; Atlanta, GA URL: https://acrabstracts.org/abstract/ polypharmacy-and-potentially-inappropriate-medication-use-in-young-versus-older-adults-with-sle/ [doi: 10.21203/rs.2.10158/v4]

46. Leelakanok N, Holcombe AL, Lund BC, Gu X, Schweizer ML. Association between polypharmacy and death: A systematic review and meta-analysis. J Am Pharm Assoc (2003) 2017;57(6):729-738.e10. [doi: 10.1016/j.japh.2017.06.002] [Medline: $\underline{28784299]}$

47. Manfredo Vieira S, Hiltensperger M, Kumar V, Zegarra-Ruiz D, Dehner C, Khan N, et al. Translocation of a gut pathobiont drives autoimmunity in mice and humans. Science 2018 Mar 09;359(6380):1156-1161 [FREE Full text] [doi: 10.1126/science.aar7201] [Medline: 29590047] 
48. He B, Hoang TK, Wang T, Ferris M, Taylor CM, Tian X, et al. Resetting microbiota by Lactobacillus reuteri inhibits T reg deficiency-induced autoimmunity via adenosine A2A receptors. J Exp Med 2017 Jan;214(1):107-123 [FREE Full text] [doi: 10.1084/jem.20160961] [Medline: 27994068]

49. Mu Q, Kirby J, Reilly CM, Luo XM. Leaky Gut As a Danger Signal for Autoimmune Diseases. Front Immunol 2017;8:598 [FREE Full text] [doi: 10.3389/fimmu.2017.00598] [Medline: 28588585]

50. Hevia A, Milani C, López P, Cuervo A, Arboleya S, Duranti S, et al. Intestinal dysbiosis associated with systemic lupus erythematosus. mBio 2014 Sep 30;5(5):e01548-e01514. [doi: 10.1128/mBio.01548-14] [Medline: 25271284]

51. NIH Integrative Human Microbiome Project. URL: https://www.hmpdacc.org/ihmp/overview/ [accessed 2008-05-20]

52. Jolly M, Katz P. Systemic lupus erythematosus: The promise of PROMIS - is it ready for prime time in SLE? Nat Rev Rheumatol 2017 Aug;13(8):453-454. [doi: 10.1038/nrrheum.2017.100] [Medline: 28660907]

53. Mahieu M, Yount S, Ramsey-Goldman R. Patient-Reported Outcomes in Systemic Lupus Erythematosus. Rheum Dis Clin North Am 2016 May;42(2):253-263 [FRE Full text] [doi: 10.1016/j.rdc.2016.01.001] [Medline: 27133488]

54. More People Search for Health Online. nbcnews.com. URL: http://www.nbcnews.com/id/3077086/t/ more-people-search-health-online/\#.Xzwi S2ZOrJ [accessed 2020-09-28]

55. Mobile Fact Sheet. URL: https://www.pewresearch.org/internet/fact-sheet/mobile/ [accessed 2020-09-20]

\author{
Abbreviations \\ BPI-SF: Brief Pain Index-Short Form \\ CNS: central nervous system \\ FACIT-F: Functional Assessment of Chronic Illness Therapy-Fatigue \\ HIPAA: Health Insurance Portability and Accountability Act \\ HRQoL: health-related quality of life \\ ITT: intention to treat \\ LupusQoL: Lupus Quality of Life \\ PP: per protocol \\ PROMs: patient-reported outcome measures \\ SLE: systemic lupus erythematosus \\ SLEDAI: Systemic Lupus Erythematosus Disease Activity Index \\ SRI: SLE Responder Index
}

Edited by G Eysenbach; submitted 26.08.20; peer-reviewed by W Gordon, A Azzam; comments to author 17.09.20; revised version
received 28.09.20; accepted 30.09.20; published 20.10.20
Please cite as:
Khan F, Granville N, Malkani R, Chathampally Y
Health-Related Quality of Life Improvements in Systemic Lupus Erythematosus Derived from a Digital Therapeutic Plus Tele-Health
Coaching Intervention: Randomized Controlled Pilot Trial
J Med Internet Res 2020;22(10):e23868
URL: $\underline{\text { https://www.jmir.org/2020/10/e23868 }}$
doi: $\underline{10.2196 / 23868}$
$P M I D: \underline{33079070}$

(CFaiz Khan, Nora Granville, Raja Malkani, Yash Chathampally. Originally published in the Journal of Medical Internet Research (http://www.jmir.org), 20.10.2020. This is an open-access article distributed under the terms of the Creative Commons Attribution License (https://creativecommons.org/licenses/by/4.0/), which permits unrestricted use, distribution, and reproduction in any medium, provided the original work, first published in the Journal of Medical Internet Research, is properly cited. The complete bibliographic information, a link to the original publication on http://www.jmir.org/, as well as this copyright and license information must be included. 